\title{
Asymptotic Comparison of the Solutions of Linear Time-Delay Systems with Point and Distributed Lags with Those of Their Limiting Equations
}

\author{
M. De la Sen \\ Department of Electricity and Electronics, Faculty of Science and Technology, \\ Institute of Research and Development of Processes (IIDP), Leioa (Bizkaia), P.O. Box. 644, \\ 48080 Bilbao, Spain \\ Correspondence should be addressed to M. De la Sen, manuel.delasen@ehu.es
}

Received 25 November 2008; Accepted 6 February 2009

Recommended by John Rassias

This paper investigates the relations between the particular eigensolutions of a limiting functional differential equation of any order, which is the nominal (unperturbed) linear autonomous differential equations, and the associate ones of the corresponding perturbed functional differential equation. Both differential equations involve point and distributed delayed dynamics including Volterra class dynamics. The proofs are based on a Perron-type theorem for functional equations so that the comparison is governed by the real part of a dominant zero of the characteristic equation of the nominal differential equation. The obtained results are also applied to investigate the global stability of the perturbed equation based on that of its corresponding limiting equation.

Copyright (C) 2009 M. De la Sen. This is an open access article distributed under the Creative Commons Attribution License, which permits unrestricted use, distribution, and reproduction in any medium, provided the original work is properly cited.

\section{Introduction}

Time-delay dynamic systems are an interesting field of research in dynamic systems and functional differential equations because of intrinsic theoretical interest because the formalism lies in that of functional differential equations, then infinite dimensional, and because of the wide range of applicability in modelling of physical systems, like transportation systems, queuing systems, teleoperated systems, war/peace models, biological systems, finite impulse response filtering, and so forth [1-4]. Important particular interest has been devoted to stability, stabilization, and model-matching of control systems where the object to be controlled possess delayed dynamics and the controller is synthesized incorporating delayed dynamics or its structure may be delay-free (see, e.g., [1, 4-14]). The properties are formulated as either being independent of or dependent on the sizes of the delays. 
An intrinsic problem which increases the analysis complexity is the presence of infinitely many characteristic zeros because of the functional nature of the dynamics. This fact generates difficulties in the closed-loop pole-placement problem compared to the delayfree case [14], as well as in the stabilization problem [2, 4-6, 8-11, 13, 15-20], including the case of singular time-delay systems where the solution is sometimes nonunique, and impulsive, because of the dynamics associated to a nilpotent matrix, [15]. The properties of the associated evolution operators have been investigated in $[2,6,11]$. Interesting recent results on infinite dimensional Banach spaces are given in [21-24]. In particular, the existence of periodic solutions of semilinear evolution equations with time lags is investigated in [21]. In [22], a class of linear impulsive periodic systems with time-varying generating operators on a Banach space is considered. The set of impulsive periodic motion controllers that are robust to parameter drift are synthesized for a given periodic motion. The research in [23] is devoted to investigate the existence and the global asymptotic stability of a periodic PCmild solution for the $T$-periodic logistic system with time-varying generating operators and $T$-0-periodic impulsive perturbations on Banach spaces. In [24], a close problem is solved based on a generalized Gronwall's lemma. In [25], the robust stability of a variational control problem is solved by providing the stability radius. Also, the approximation properties of the homogeneous system associated with a class of linear elliptic differential equations with periodic coefficients is investigated in [26].

This paper is devoted to obtain results relying on a comparison and an asymptotic comparison of the eigensolutions between a nominal (unperturbed) functional differential equation involving wide classes of delays and a perturbed version (describing the current dynamics) with some appropriate assumptions smallness in the limit on the perturbed functional differential equation. The nominal equation is defined as the limiting equation of the perturbed one since the parameters of the last one converge asymptotically to those of its limiting counterpart. The problem is of interest in practice since the perturbations related to a nominal model in dynamic systems very often occur during the transients while they are asymptotically vanishing in the steady-state or, in the most general worst case, they grow at a smaller rate than the solution of the nominal differential equation. In this context, the nominal differential equation may be viewed as the limiting equation of the perturbed one. The comparison between the solutions of the limiting differential equation and those of the perturbed one based on Perron-type results have been studied classically for ordinary differential equations and, more recently, for the case of functional equations [2729]. Particular functional equations of interest in real-life problems are those involving both point and distributed delays, the last ones potentially include Volterra-type terms, $[2,5-7,30]$.

\section{Notation}

The following sets are used through the manuscript:

$$
\begin{gathered}
\mathbf{R}_{0+}:=\mathbf{R}_{+} \cup\{0\}, \quad \mathbf{R}_{+}:=\{z \in \mathbf{R}: z>0\}, \quad \mathbf{R}_{0-}:=\mathbf{R}_{-} \cup\{0\}, \quad \mathbf{R}_{-}:=\{z \in \mathbf{R}: z<0\}, \\
\mathbf{C}_{0+}:=\{z \in \mathbf{C}: \operatorname{Re} z \geq 0\}, \quad \mathbf{C}_{0-}:=\{z \in \mathbf{C}: \operatorname{Re} z \leq 0\}, \quad \mathbf{C}_{-}:=\{z \in \mathbf{C}: \operatorname{Re} z<0\}, \\
\mathbf{Z}_{0+}:=\mathbf{Z}_{+} \cup\{0\}, \quad \mathbf{Z}_{+}:=\{z \in \mathbf{Z}: z>0\},
\end{gathered}
$$

where $\mathbf{R}, \mathbf{C}$, and $\mathbf{Z}$ are the sets of real, complex and integer numbers, respectively, The complex imaginary unity is $\mathbf{i}=\sqrt{-1}$. 
A finite subset of $j$ consecutive positive integers starting with 1 is denoted by $\bar{j}:=$ $\{1,2, \ldots, j\}$. The set $\mathbf{R}_{-h}:=[-h, 0) \cup \mathbf{R}_{0+}$ will be used to define the solution of functional differential equations on $\mathbf{R}_{0+}$ including its initial condition in $[-h, 0]$.

$C^{(i)}\left(\mathbf{R}_{0+}, \mathbf{R}_{0+}^{m}\right)$ is the set of m-vector real functions of class $C^{(i)}$ and definition domain $\mathbf{R}_{0+}$ and $\mathrm{PC}^{(i)}\left(\mathbf{R}_{0+}, \mathbf{R}_{0+}^{m}\right)$ is the set of m-vector real functions in $C^{(i-1)}\left(\mathbf{R}_{0+}, \mathbf{R}_{0+}^{m}\right)$ whose $i$ th derivative is piecewise continuous. Similar sets of functions are defined when the ranges are complex as $C^{(i)}\left(\mathbf{R}_{0+}, \mathbf{C}_{0+}^{m}\right)$ and $\mathrm{PC}^{(i)}\left(\mathbf{R}_{0+}, \mathbf{C}_{0+}^{m}\right)$.

For the delayed system, $T:[0, \infty) \rightarrow L(X)$ is the inverse Laplace transform of the resolvent mapping $\widehat{T}(s)$, which is holomorphic where it exists, with $X$ being the real Banach space of $n$-vector real functions endowed with the supremum norm on their definition domain defined for any such a complex or real vector function $\phi$ of definition domain $D$ by $|\phi|_{\alpha}=\sup _{\tau \in D}\left(\|\phi(\tau)\|_{\alpha}\right) \cdot\|\cdot\|_{\alpha}$ denoting any vector or induced matrix norm, that is, $\alpha=1$ for the $\ell_{1}$-norm, $\alpha=\infty$ for the $\ell_{\infty}$-norm, and so forth. Similar notations are used for the corresponding matrix-induced norms. In particular, $\alpha=2$ stands for $\ell_{2}$ (or spectral) vector and corresponding induced matrix norms, which coincides with the Euclidean norm for vectors. The Euclidean (or Froebenius) norm is denoted by the unsubscripted symbol $\|\cdot\|$ so that $\|\cdot\|=\|\cdot\|_{2}$ for vectors but not for matrices. In the case of vectors, the Euclidean norm coincides with its $\ell_{2}$-norm.

The unsubscripted symbol $|\cdot|$ is used for absolute values of real, complex, and integer numbers, as usual. It is said that the delays associated with Volterra-type dynamics are infinitely distributed because the contribution of the delayed dynamics is made under an integral over $[0, \infty)$ as $t \rightarrow \infty$, that is, $x\left(t-\tau-h_{i}^{\prime}\right)$ acts on the dynamics of $x(t)$ from $\tau=0$ to $\tau=t$ for finite $t$ and as $t \rightarrow \infty$.

$\operatorname{Dom}(\mathrm{H})$ is the definition domain of the operator $\mathrm{H}$ and $\operatorname{sp}(A)$ is the spectrum (i.e., the set of distinct eigenvalues) of the square matrix $A$. The matrix measure of the normdependent complex-valued matrix $A$ is defined by $\kappa_{\alpha}(A):=\lim _{\delta \rightarrow 0^{+}}\left(\left\|I_{n}+\delta A\right\|_{\alpha}-\delta\right) / \delta \geq$ $\operatorname{Re} \lambda_{i}(A)$, for all $\lambda_{i} \in \operatorname{sp}(A)$.

Also, $\neg, \vee, \wedge$ are logic symbols for negation, disjunction, and conjuction of logic propositions.

\section{Problem Statement and Basic First Results}

Consider the following linear nominal functional differential systems with point and, in general, both Volterra-type and finite distributed delays:

$$
\begin{gathered}
\dot{x}(t)=\mathrm{L} x_{t} \equiv \sum_{i=0}^{m} A_{i} x\left(t-h_{i}\right)+\sum_{i=0}^{m^{\prime}} \int_{0}^{t} d \alpha_{i}(\tau) A_{\alpha_{i}} x\left(t-\tau-h_{i}^{\prime}\right)+\sum_{i=m^{\prime}+1}^{m^{\prime}+m^{\prime \prime}} \int_{t-h_{i}^{\prime}}^{t} d \alpha_{i}(t-\tau) A_{\alpha_{i}} x(\tau), \\
\dot{x}(t)=L x_{t}+f\left(t, x_{t}\right) \\
\equiv \sum_{i=0}^{m} A_{i} x\left(t-h_{i}\right)+\sum_{i=0}^{m^{\prime}} \int_{0}^{t} d \alpha_{i}(\tau) A_{\alpha_{i}} x\left(t-\tau-h_{i}^{\prime}\right) \\
+\sum_{i=m^{\prime}+1}^{m^{\prime}+m^{\prime \prime}} \int_{t-h_{i}^{\prime}}^{t} d \alpha_{i}(t-\tau) A_{\alpha_{i}} x(\tau)+f\left(t, x_{t}\right)
\end{gathered}
$$




$$
\begin{aligned}
f\left(t, x_{t}\right)= & \sum_{i=0}^{m} \tilde{A}_{i}(t) x\left(t-h_{i}\right)+\sum_{i=0}^{m^{\prime}} \int_{0}^{t} d \alpha_{i}(\tau) \tilde{A}_{\alpha_{i}}(t) x\left(t-\tau-h_{i}^{\prime}\right) \\
& +\sum_{i=m^{\prime}+1}^{m^{\prime}+m^{\prime \prime}} \int_{t-h_{i}^{\prime}}^{t} d \alpha_{i}(t-\tau) \widetilde{A}_{\alpha_{i}}(\tau) x(\tau)+f_{0}\left(t, x_{t}\right) .
\end{aligned}
$$

Equation (2.1) is the limiting equation of the perturbed equation (2.2), subject to (2.3), for $f\left(t, x_{t}\right) \rightarrow 0$ as $t \rightarrow \infty$ under the following technical hypothesis.

H.1: The initial conditions of both differential equations (2.1) and (2.2) are real n-vector functions $\phi \in C_{e}(-h)$ where $C_{e}(-h):=\left\{\phi=\phi_{1}+\phi_{2}: \phi_{1} \in C(-h), \phi_{2} \in B^{0}(-h)\right\}, \phi(0)=x_{0}$, with $C(-h):=\left\{C^{0}([-h, 0], X)\right\}$; that is, the set of continuous mappings from $[-h, 0]$ into the Banach space $X$ with norm $\bar{\phi}_{\alpha}:=|\phi|_{\alpha}=\operatorname{Sup}\left\{\|\phi(t)\|_{\alpha}:-h \leq t \leq 0\right\} ;\|\cdot\|$ denoting the Euclidean norm of vectors in $C^{n}$ and matrices in $C^{n \times n}$, and $B^{0}(-h):=\{\phi:[-h, 0] \rightarrow X\}$ is the set of realbounded vector functions on $X$ endowed with the supremum norm having support of zero measure. Roughly speaking, $\phi \in B^{0}(-h)$ if and only if it is almost everywhere zero except at isolated discontinuity points within $[-h, 0]$ where it is bounded. Thus, $\phi \in C_{e}(-h)$ if and only if it is almost everywhere continuous in $[-h, 0]$ except possibly on a set of zero measure of bounded discontinuities. $C_{e}(-h)$ is also endowed with the supremum norm since $\phi=\phi_{1}+\phi_{2}$, some $\phi_{1} \in C(-h), \phi_{2} \in B^{0}(-h)$ for each $\phi \in C_{e}(-h)$. In the following, the supremum norms on $L(X)$ are also denoted with $|\cdot|$.

Close spaces of functions are $C\left(\mathbf{R}_{-h}\right):=C\left([-h, \infty), \mathbf{C}^{n}\right)$ which is the Banach space of continuous functions from $[-h, \infty)$ into $\mathbf{C}^{n}$ endowed with the norm $|\phi|_{\alpha}=$ $\sup _{-h \leq \tau<\infty}\left(\|\phi(\tau)\|_{\alpha}\right)$; for all $\phi \in C_{e}(-h):=C\left([-h, 0), \mathbf{C}^{n}\right)$ being an initial condition, for some given vector norm $\|\cdot\|_{\alpha}$. Note that for $t \in \mathbf{R}_{0_{+}}$, the solution which satisfies (2.2), subject to (2.3), is in $C_{e}\left(\mathbf{R}_{0+}\right):=C\left(\mathbf{R}_{0+}, \mathbf{C}^{n}\right)$, the Banach space of continuous functions from $\mathbf{R}_{0+}$ into $\mathbf{C}^{n}$ which satisfies (2.2)-(2.3), $\forall \phi \in C_{e}(-h)$, endowed with $|\phi|_{\alpha}=\sup _{0 \leq \tau<\infty}\left(\|\phi(\tau)\|_{\alpha}\right)$.

Thus, $L: C_{e}\left(\mathbf{R}_{-h}\right) \rightarrow \mathbf{C}^{n}$ is a bounded linear functional defined by the right-hand-side of (2.1).

H.2: All the operators $A_{k}(0 \leq k \leq m), A_{\alpha_{k}}\left(0 \leq k \leq m^{\prime}+m^{\prime \prime}\right)$ are in $L(X):=L(X, X)$, the set of linear operators on $X$, of dual $X^{*}$ and $h_{k}$, and $h_{\ell}^{\prime}\left(k=1,2, \ldots, m ; \ell=0,1, \ldots, m^{\prime}+m^{\prime \prime}\right)$ are nonnegative constants with $h_{0}=h_{0}^{\prime}=0$ and $h:=\operatorname{Max}\left(\operatorname{Max}_{1 \leq i \leq n}\left(h_{i}\right), \operatorname{Max}_{1 \leq i \leq m^{\prime}+m^{\prime \prime}}\left(h_{i}^{\prime}\right)\right)$.

H3: The linear operators $A_{\alpha_{i}} \in L(X)$, with abbreviated notation $A_{\alpha_{0}}=A_{\alpha}$, are closed and densely defined linear operators with respective domain and range $D\left(A_{\alpha_{i}}\right)$ and $R\left(A_{\alpha_{i}}\right) \subset X\left(i=0,1, \ldots, m^{\prime}+m^{\prime \prime}\right)$. The functions $\alpha_{i} \in C^{0}([0, \infty), \mathbf{C}) \cap B V_{\text {loc }}\left(\mathbf{C}_{+}\right)(i=$ $\left.0,1, \ldots, m^{\prime}\right)$ and $\alpha_{i} \in C^{0}([-h, 0), C)\left(i=0,1, \ldots, m^{\prime}+m^{\prime \prime}\right)$ being everywhere differentiable with possibly bounded discontinuities on subsets of zero measure of their definition domains with $\int_{0}^{\infty} e^{v t}\left|d \alpha_{i}(\mathrm{t})\right|<\infty$ some nonnegative real constant $v\left(i=0,1, \ldots, m^{\prime}\right)$. If $\alpha_{i}(\cdot)$ is a matrix function $\alpha_{i}:[0, \infty] \times X^{*} \rightarrow L\left(X, X^{*}\right)$ then it is in $C^{0}\left([0, \infty), C^{\mathbf{n} \times \mathbf{n}}\right) \cap B V_{\text {loc }}\left(C_{+}^{\mathbf{n} \times \mathbf{n}}\right)$ with $\int_{0}^{\infty} e^{\nu t}\left|d \alpha_{i}(t)\right|<\infty$ and its entries being everywhere time-differentiable with possibly bounded discontinuities on a subset of zero measure of their definition domains.

H.4: It is assumed that $f_{0}: \mathbf{R}_{0+} \times C\left(\mathbf{R}_{-h}\right) \rightarrow \mathbf{C}^{n}$ and $h_{0}=h_{0}^{\prime}=0$,

$$
x_{t}^{C\left(\mathbf{R}_{-h}\right)}:= \begin{cases}x:[-h, \tau) \longrightarrow X, & \tau \leq t \\ 0, & \tau>t\end{cases}
$$


satisfying $x(t)=\phi(t)$, for all $t \in[-h, 0]$, is a string of the solution of (2.2)-(2.3). Other strings of the solution trajectory of interest in this manuscript are $x_{t}^{C\left(\mathbf{R}_{0^{+}}\right)}$which point-wise defined by $x(t)$ within the interval $[0, t]$ and zero, otherwise, and subject to the constraint $x_{t}=\phi_{t}$ within $[\max (-h, t-h), t], \forall t \in \mathbf{R}_{0+}$ and being zero outside this interval. Finally, $x_{t}$ denotes the solution string within $[t-h, t]$ point-wise defined by the solution $x(t)$ to (2.2)-(2.3) for each $t \in \mathbf{R}_{-h}$ being zero outside $[t-h, t]$ and subject to the constraint $x_{t}=\phi_{t}$ within $[\max (-h, t-h), t]$ for any real $t \leq h$ and being zero outside this interval.

$A_{i}$ and $A_{\alpha_{k}}$ and $\tilde{A}_{i}:[0, \infty) \rightarrow \mathrm{C}^{n \times n}$ and $\tilde{A}_{i}:[0, \infty) \rightarrow \mathrm{C}^{n \times n}(k=0,1, \ldots, m ; k=$ $\left.0,1, \ldots, m^{\prime}+m^{\prime \prime}\right)$ belong to the spaces of constant real matrices and real matrix functions, respectively. The last ones are also unbounded operators on a Banach space of n-vector real functions $x \in X$ endowed with the supremum norm where the vectors of point and distributed constant delays are: $\widehat{h}:=\left(0, h_{1}, h_{2}, \ldots, h_{m}\right)$ and $\widehat{h}^{\prime}=\left(\widehat{h}_{1}^{T}: \widehat{h}_{2}^{\prime T}\right)^{T}:=$ $\left(0, h_{1}^{\prime}, h_{2}^{\prime}, \ldots, h_{m^{\prime}}^{\prime}: h_{m^{\prime}+1}^{\prime}, h_{m^{\prime}+2^{\prime}}^{\prime}, \ldots, h_{m^{\prime}+m^{\prime \prime}}^{\prime}\right)^{T}$, respectively, with $h_{i} \geq 0$ and $h_{k}^{\prime} \geq 0(k=$ $\left.1,2, \ldots, m^{\prime}+m^{\prime \prime}\right)$ and being, respectively, point and distributed delays, with $h_{0}=h_{k}^{\prime}=0, A_{0} \equiv$ $A, A_{\alpha}$ and $\alpha_{0}(\cdot) \equiv \alpha(\cdot)$. The first $m^{\prime}$ distributed delays are associated with Volterra-type dynamics. In other words, the infinitely distributed delays give contributions $\int_{0}^{t} d \alpha_{i}(\tau) A_{\alpha_{i}} x(t-$ $\left.\tau-h_{i}^{\prime}\right)$ with finite real constants $h_{i}^{\prime}$ with $\left(i=1,2, \ldots, m^{\prime}\right)$ to $\dot{x}(t)$ which are point delays under the integral symbol. The functions $\alpha_{i}:[0, \infty) \rightarrow \mathrm{C}$ and $\alpha_{k}:\left\lfloor 0, h_{k}^{\prime}\right\rfloor \rightarrow \mathrm{C}$ are continuously differentiable real functions within their definition domains except possibly on sets of zero measure where the time-derivatives have bounded discontinuities. All or some of the $\alpha_{i}(\cdot)$ may be alternatively matrix functions $\alpha_{i}:[0, t] \rightarrow C^{n \times n}\left(i=0,1, \ldots, m^{\prime}\right)$ for $t \in \mathbf{R}_{+}$and $\alpha_{i}:\left\lfloor 0, h_{k}^{\prime}\right\rfloor \rightarrow C^{n \times n}\left(i=m^{\prime}+1, m^{\prime}+2 \ldots, m^{\prime}+m^{\prime \prime}\right)$ with $\alpha_{i}(0)=0 ; i=0,1, \ldots, m^{\prime}+m^{\prime \prime}$. On the other hand, the perturbation vector function $f\left(t, x_{t}\right)$ in (2.2), defined in (2.3), with respect to the limiting (2.1), is defined by the function $f: \mathbf{R}_{0+} \times C\left(\mathbf{R}_{-h}\right) \rightarrow \mathbf{C}^{n \times n}$ which describes a perturbed dynamics associated with the delays plus a perturbation function $f_{0}: \mathbf{R}_{0+} \times C\left(\mathbf{R}_{-h}\right) \rightarrow \mathbf{C}^{n \times n}$ which is not included in the remaining terms of the function $f$ in (2.3). Note that both the delayed differential systems (2.2)-(2.3) and its limiting version (2.1) are very general since it includes point-delayed dynamics, like, for instance, in typical war/peace models or the so-called Minorski's problem appearing when controlling the lateral dynamics of a ship [2]. It also includes real constants $h_{i}^{\prime}\left(i=0,1, \ldots, m^{\prime}\right)$, with $h_{0}^{\prime}=0$, associated with infinitely distributed delayed contributions to the dynamics. Such delays are relevant, for instance, in viscoelastic fluids, electrodynamics, and population growth $[1,5,8]$. In particular, an integro-differential Volterra-type term is also included through $h_{0}^{\prime}=0$. Apart from those delays, the action of finite-distributed delays characterized by real constants $h_{i}^{\prime}\left(i=0,1, \ldots, m^{\prime}+m^{\prime \prime}\right)$ is also included in the limiting equation (2.1) and in (2.3). That kind of delays is well known, for instance, in econometric models related to production rate [8]. The integrability of the $\alpha_{i}(\cdot)$-functions (or matrix functions) on $\left[t-h_{i}^{\prime}, t\right]$ follows since their definition domain is bounded. The technical hypothesis $\mathrm{H} 1-\mathrm{H} 4$ guarantee the existence and uniqueness of the solution in $C\left(\mathbf{R}_{0+}\right):=C\left(\mathbf{R}_{0+}, \mathrm{C}^{n}\right)$ of the functional differential systems (2.1) and (2.2)-(2.3) for each given initial condition $\phi \in C_{e}(-h)$.

Take Laplace transforms in (2.1) by using the convolution theorem and the relations $d \alpha(\tau)=\dot{\alpha}(\tau) d \tau$. It follows that $d \widehat{\alpha}_{i}(s)=s \widehat{\alpha}_{i}(s)-\alpha_{i}(0)$, where $\widehat{f}(s):=\operatorname{Lap} f(t)$ denotes the Laplace transform of $f(t)$. Thus, the unique solutions of both the limiting (2.1) and that of (2.2)-(2.3) in $C\left(\mathbf{R}_{0+}\right)$, subject to (2.3); for all $t \in \mathbf{R}_{0+}$, for the same given initial conditions $\phi \in C_{e}(-h)$ are, respectively, defined by 


$$
\begin{gathered}
y(t)=T(t, 0) x\left(0^{+}\right)+\int_{-h}^{0} T(t, \tau) \phi(\tau) U(\tau) d \tau \\
x(t)=T(t, 0) x\left(0^{+}\right)+\int_{-h}^{0} T(t, \tau) \phi(\tau) U(\tau) d \tau+\int_{0}^{t} T(t, \tau) f\left(\tau, x_{\tau}\right) d \tau,
\end{gathered}
$$

where $U(t)$ is the unit step (Heaviside) function and $T(t, \tau)$ is the evolution operator, $[2,6,31]$, of the linear (2.1) whose Laplace transform, everywhere it exists, is given by the resolvent:

$$
\begin{aligned}
\widehat{T}(s):=\operatorname{Lap} T(t, 0)= & {\left[s\left(I_{n}-\sum_{i=0}^{m^{\prime}} \widehat{\alpha}_{i}(s) A_{\alpha_{i}} e^{-h_{i}^{\prime} s}-\sum_{i=m^{\prime}+1}^{m^{\prime}+m^{\prime \prime}} \widehat{\alpha}_{i}(s) A_{\alpha_{i}}\left(1-e^{-h_{i}^{\prime} s}\right)\right)\right.} \\
& \left.-\sum_{i=0}^{m} A_{i} e^{-h_{i} s}+\alpha(0) A_{\alpha}+\sum_{i=0}^{m^{\prime}} \alpha_{i}(0) A_{\alpha_{i}} e^{-h_{i}^{\prime} s} \sum_{i=m^{\prime}+1}^{m^{\prime}+m^{\prime \prime}} \alpha_{i}(0) A_{\alpha_{i}}\left(1-e^{-h_{i s}^{\prime}}\right)\right]^{-1} .
\end{aligned}
$$

As usual, it is said though the manuscript that (2.1) is the limiting equation of (2.2)-(2.3) irrespective of the fact that $f\left(t, x_{t}\right)$ converges or not to zero as $t \rightarrow \infty$. The evolution operator is a convolution operator so that $T(t, \tau)=T(t-\tau, 0)=T(t-\tau)$ if the Volterra-type dynamics is zero or if the associate differentials in the Riemann-Stieltjes integrals $d \alpha_{i}(t)=x_{i} d t$ with $x_{i}$ being real constants. In this case, the limiting linear functional differential equation is, furthermore, time-invariant. Note that the limiting (2.1) is guaranteed to be globally exponentially uniformly stable if and only if $\widehat{T}(s)$ exists within some region including properly the right-complex plane. In other words, if it is compact for $\operatorname{Re} s>-\alpha_{0}$, for some $r$ constant $\alpha_{0} \in \mathbf{R}$ located to the right of all the real parts of all the zeros of $\operatorname{det} \widehat{T}^{-1}(s)$ (also often called the characteristic zeros of the limiting (2.2) or, simply, its eigenvalues), since then all the entries of its Laplace transform $T(t)$ decay with exponential rate on $\mathbf{R}_{0_{+}}$for $\phi \in C_{e}(-h)$ and then $|x(t)|$ decays with exponential rate on $\mathbf{R}_{+}$. The main result addressed in $[2,7-10]$ relies on the investigation of the global uniform exponential stability of (1). The stability of the limiting system (2.1) is investigated in $[5,6]$, provided that any auxiliary system formed with any of the additive parts of the dynamics of (2.1), has such a property and provided that an impulsive-solution-dependent input exists. The compactness of the relevant input-output and input-state operators under forcing external inputs and impulsive forcing terms is also investigated in [6]. The basic mathematical tool used in those papers is that the unique solution of the homogeneous (2.1) for each function of initial conditions $\phi \in C_{e}(-h)$ may be equivalently written in infinitely many cases by first rewriting (2.1) by considering different "auxiliary" reference homogeneous systems plus additional terms considered as forcing actions. The objective of this paper is to compare the solutions (2.1), (2.2), subject to (2.3), of the limiting and current functional differential equations (2.1) and (2.2) by using a Perron-type result using a similar technique as that used in [28]. The subsequent theorem is a generalization of a classical Perron-type theorem for ordinary differential equations to (2.2), subject to (2.3) compared to (2.1) (see [1, Chapter IV, Theorem 5] and [28, Theorem 1.1] for functional differential equations which include several kinds of delays such as point and distributed delays and Volterra-type dynamics with infinite delays. The result extends the perturbation term to include constant upper-bounding terms in the perturbation functional (2.3) and characteristic zeros of the limiting (2.2) (i.e., zeros det $\widehat{T}^{-1}(s)$ ) of multiplicity greater than unity (being degenerated or non-degenerated) in the limiting dynamics defined by (2.1). 
Theorem 2.1. Let $x$ be a solution of (2.2), subject to (2.3), on $\mathbf{R}_{0+}$ subject to initial conditions $\phi \in$ $C_{e}(-h)$ such that

$$
\left|f\left(t, x_{t}\right)\right|_{\alpha} \leq \gamma_{\alpha}(t)\left|x_{t}\right|_{\alpha}+K_{0 \alpha}, \quad t \in \mathbf{R}_{0+}
$$

for some norm-dependent $K_{0 \alpha} \in \mathbf{R}_{0+}$ where $\gamma_{\alpha} \in C^{(0)}\left(\mathbf{R}_{0^{+}}, \mathbf{R}_{0+}\right)$ is also norm-dependent and satisfies $\int_{t}^{t+1}\left(\left(t^{\vartheta_{k}-1}\right) /\left(\vartheta_{k} !\right)\right) e^{\beta \sigma_{k} t} \gamma_{\alpha}(s) d s \rightarrow 0$ as $t \rightarrow \infty$, where $\beta=0$ if there is no Volterra term in (2.1) and $\beta=1$, otherwise, and $\sigma_{k}$ are the real parts of the zeros of $\operatorname{det} \widehat{T}^{-1}(s)$ of the limiting (2.2) with respective multiplicities $\vartheta_{k}$. Then, the following properties hold:

(i)

$$
\lim _{t \rightarrow \infty} \int_{t}^{t+1} g_{\alpha}(s)\left(\left|\frac{f\left(s, x_{s}\right)}{x_{s}}\right|_{\alpha}-K_{0 \alpha}\right) d s=0,
$$

where $g_{\alpha} \in P C^{(0)}\left(\mathbf{R}_{0+}, \mathbf{R}_{0+}\right)$ is an indicator function defined by

$$
g_{\alpha}(t)= \begin{cases}0 & \text { if } \gamma_{0 \alpha}(t)=\left|\frac{f\left(t, x_{t}\right)}{x_{t}}\right|_{\alpha}-K_{0 \alpha} \leq 0 \\ 1 & \text { otherwise. }\end{cases}
$$

(ii) The real numbers $\mu_{k}=\mu_{k}(x)=\lim _{t \rightarrow \infty}\left(\log \left|x_{t}\right|\right) / t^{k}$ exist and are norm-independent and finite, for all $k \geq k_{1}$ and some integer $k_{1} \geq 1$ with $\mu_{k}=0, \forall k>k_{1}$ or $\lim _{t \rightarrow \infty} e^{b t} x(t)=0, b \in \mathbf{R}$. If $\mu_{k_{1}} \geq 0$ or if (2.8) holds with $K_{0 \alpha}=0$, then either $\mu_{k_{1}}$ it is the real part of a zero of $\operatorname{det} \widehat{T}^{-1}(s)$, for which the resolvent $\widehat{T}(s)$ trivially exists and it is bounded, or $\lim _{t \rightarrow \infty} e^{b t} x(t)=0$, for all $b \in \mathbf{R}$.

(iii) Assume that all the zeros of $\operatorname{det} \widehat{T}^{-1}(s)$ have real negative parts and (2.8) holds only for some constants $K_{0} \mathbf{R}_{+}$. Then, either the limits $\mu_{k}=\mu_{k}(x)=\lim _{t \rightarrow \infty}\left(\log \left|x_{t}\right|\right) / t^{k}=0$ exist, $\forall k \geq k_{1}$, some integer $k_{1} \geq 1$, and furthermore, $\mu_{1}$ is not trivially the real part of a characteristic zero of (2.2), or $\lim _{t \rightarrow \infty} e^{b t} x(t)=0, \forall b \in \mathbf{R}$.

Proof. (i) From (2.8), $\gamma_{\alpha(t)} \geq \max \left(0,\left|f\left(t, x_{t}\right) / x_{t}\right|_{\alpha}-K_{0 \alpha}\right)=g_{\alpha}(t)\left(0,\left|f\left(t, x_{t}\right) / x_{t}\right|_{\alpha}-K_{0 \alpha}\right) \geq 0$ so that

$$
\begin{aligned}
0 & =\lim _{t \rightarrow \infty} \int_{t}^{t+1} \gamma_{\alpha}(s) d s \geq \limsup _{t \rightarrow \infty} \int_{t}^{t+1} g_{\alpha}(s)\left(\left|\frac{f\left(s, x_{s}\right)}{x_{s}}\right|_{\alpha}-K_{0 \alpha}\right) d s \geq 0 \\
& \Longrightarrow \lim _{t \rightarrow \infty} \int_{t}^{t+1} g_{\alpha}(s)\left(\left|\frac{f\left(s, x_{s}\right)}{x_{s}}\right|_{\alpha}-K_{0 \alpha}\right) d s=0
\end{aligned}
$$

and property (i) has been proved. 
(ii) From (2.8), $\left|f\left(t, x_{t}\right)\right|_{\alpha}=\gamma_{\alpha}(t)\left|x_{t}\right|_{\alpha}+K_{0 \alpha}-\omega_{\alpha}(t), t \in \mathbf{R}_{0+}$, some $\omega_{\alpha} \in P C^{(0)}\left(\mathbf{R}_{0+}, \mathbf{R}_{0+}\right)$. Then, one gets from (2.6)-(2.7),

$$
\begin{aligned}
\|x(t)\|_{\alpha} \leq K_{1}(\alpha) \frac{t^{\nu-1}}{v !}\left[e^{\mu t}\left\|x\left(0^{+}\right)\right\|_{\alpha}+\left|\frac{e^{\mu t}\left(1-e^{\mu h}\right)}{\mu}\right|\left(|\phi|_{\alpha}+K_{0 \alpha}\right)\right. \\
\left.\quad+\beta \max \left(1, e^{\mu t}\right) \sup _{0 \leq \tau \leq t}\left(\left|x_{t}\right|_{\alpha}\right)\left(\sum_{l=0}^{j_{t}} \int_{\ell}^{\ell+1} \gamma_{\alpha}(s) d s+\int_{j_{t}}^{t} \gamma_{\alpha}(s) d s\right)\right]
\end{aligned}
$$

from the limiting hypothesis on the integral of the function $\gamma_{\alpha}$, for any arbitrary small real norm-dependent constant $\varepsilon_{\alpha} \in \mathbf{R}_{+}$, there exists a finite $t_{0} \in \mathbf{Z}_{+}$( only for a simple constructive proof easily extendable to $t_{0} \in \mathbf{R}_{+}$), dependent on $\varepsilon_{\alpha}$ and the given $\alpha$-norm, such that one gets from (2.12) by taking initial conditions at $t_{0}$ :

$$
\begin{gathered}
\|x(t)\| \alpha \leq K_{1}(\alpha) \frac{\left(t-t_{0}\right)^{\nu-1}}{\nu !}\left[e^{\mu\left(t-t_{0}\right)}\left\|x\left(t_{0}^{+}\right)\right\|_{\alpha}+\left|\frac{e^{\mu\left(t-t_{0}\right)}\left(1-e^{\mu h}\right)}{\mu}\right|\left(|\phi|_{\alpha}+K_{0 \alpha}\right)\right. \\
\left.+\beta \varepsilon_{\alpha}\left(1, e^{\mu t}\right) \sup _{t-t_{0} \leq \tau \leq t}\left(\left|x_{t}\right|_{\alpha}\right)\right]
\end{gathered}
$$

with $\mu$ being the real part of a characteristic zero of $\widehat{T}^{-1}(s)$ of multiplicity $v$ and $j_{t}=\max (z \in$ $\left.\mathbf{Z}_{0+}: t \geq j_{t}\right)$ is dependent on $t$. Note that, if the solution $x(t)$ is unbounded for the given initial conditions, then there exist, by construction, a finite and $t_{0} \in \mathbf{Z}_{+}$and a subsequence $x\left(t_{k}\right)$ valued at the real increasing sequence $\left\{t_{k}\right\}_{0}^{\infty}$ (then $t_{k} \rightarrow \infty$ as $k \rightarrow \infty$ ) such that $\left\|x\left(t_{k}\right)\right\|_{\alpha}=\sup _{t-t_{0} \leq \tau \leq t_{k}}\left(\left|x_{\tau}\right|_{\alpha}\right)$ so that from (2.13) and for some bounded vector function $g_{\alpha} \in$ $P C^{(0)}\left(\mathbf{R}_{0+}, \mathbf{R}_{0+}\right)$,

$$
\begin{aligned}
\left\|x\left(t_{k}\right)\right\|_{\alpha}= & \sup _{t_{k}-t_{0} \leq t \leq t_{k}}\left(\left|x_{t}\right|_{\alpha}\right) \\
\leq\left(1-\beta \varepsilon_{\alpha} K_{1}(\alpha)\right)^{-1}( & K_{1}(\alpha) \frac{\left(t_{k}-t_{0}\right)^{v-1}}{v !} \\
\times & \left.\times\left[e^{\mu t\left(t_{k}-t_{0}\right)}\left\|x\left(t_{0}^{+}\right)\right\|_{\alpha}+\left|\frac{e^{\mu\left(t_{k}-t_{0}\right)}\left(1-e^{\mu h}\right)}{\mu}\right|\left(|\phi|_{\alpha}+K_{0 \alpha}\right)\right]\right)
\end{aligned}
$$

provided that $\varepsilon_{\alpha}$ is sufficiently small to guarantee $1>\varepsilon_{\alpha} K_{1}(\alpha)$ in the case that $\beta=1$ and independently of $\varepsilon_{\alpha}$ if $\beta=0$. Furthermore, if $\mu \neq 0$ then $\mu>0$ if the solution is unbounded since, otherwise, $\sup _{t_{k}-t_{0} \leq t \leq t_{k}}\left(\left|x_{t}\right|_{\alpha}\right)$ is bounded from (2.8) which contradicts the made assumption that it is unbounded. The equivalent contrapositive proposition to the last above one is that if $\sup _{t_{k}-t_{0} \leq t \leq t_{k}}\left(\left|x_{t}\right|_{\alpha}\right)$ is uniformly bounded then $\mu \leq 0$. Equivalently, if furthermore $\mu=0$, then $v=1$ (i.e., $\mu$ is the real part of a simple real characteristic zero of $\widehat{T}^{-1}(s)$ associate with the limiting equation (2.2) or there are two simple complex conjugate ones with real part $\mu$ ). Otherwise, some unbounded lower-bound may be obtained similarly to (2.13) with the replacement of one of the plus signs in the right-hand-side terms with a minus sign affecting some unbounded term caused by $\left(t-t_{0}\right)^{v-1} / v ! \rightarrow \infty$ as $t \rightarrow \infty$ if $v \neq 1$. 
This implies that the solution is unbounded which contradicts the fact that it is bounded. Note from (2.13) that if $\mu>0$ then real increasing sequence $\left\{t_{k}\right\}_{0}^{\infty}$ of (2.13):

$$
\begin{gathered}
\sup _{t_{j}-t_{0} \leq \leq t_{k}}\left(\left|x_{t_{j}}\right|_{\alpha}\right)=K_{1}(\alpha) \frac{\left(t_{k}-t_{0}\right)^{\nu-1}}{v !}\left[e^{\mu\left(t_{k}-t_{0}\right)}\left\|x\left(t_{0}^{+}\right)\right\| \alpha+\left|\frac{e^{\mu\left(t_{k}-t_{0}\right)}\left(1-e^{\mu h}\right)}{\mu}\right|\left(|\phi|_{\alpha}+K_{0 \alpha}\right)\right. \\
\left.\quad+\beta \varepsilon_{\alpha} \sup _{t_{k}-t_{0} \leq \tau \leq t_{k}}\left(\left.\left|x_{t_{k}}\right|\right|_{\alpha}\right)\right]-g_{\alpha}\left(t_{0}, t_{k}\right) \\
\leq\left(1-\beta \varepsilon_{\alpha} K_{1}(\alpha)\right)^{-1}\left(K_{1}(\alpha) \frac{\left(t_{k}-t_{0}\right)^{\nu-1}}{\nu !}\right. \\
\times\left[e^{\mu\left(t_{k}-t_{0}\right)}\left\|x\left(t_{0}^{+}\right)\right\|_{\alpha}+\left|\frac{e^{\mu\left(t_{k}-t_{0}\right)}\left(1-e^{\mu h}\right)}{\mu}\right|\left(|\phi|_{\alpha}+K_{0 \alpha}\right)\right] \\
\left.-g_{\alpha}\left(t_{0}, t_{k}\right)\right)
\end{gathered}
$$

which takes the form $\sup _{t_{k}-t_{0} \leq t \leq t k}\left(\left|x_{t}\right|_{\alpha}\right)=\left(\left(t_{k}-t_{0}\right)^{\nu-1} / v !\right) e^{\mu\left(t_{k}-t_{0}\right)} M-g_{\alpha}\left(t_{0}, t_{k}\right)$, where $M \in$ $\mathbf{R}_{0+}$ depends on $t_{0}$ (finite), $K_{1 \alpha}, K_{0 \alpha}, \beta, \varepsilon_{\alpha},\left|\phi_{\alpha}\right|$, and the $\alpha$-norm, for some bounded vector function $g_{\alpha} \in P C^{(0)}\left(\mathbf{R}_{0+}, \mathbf{R}_{0+}\right)$ which depends on $t_{0}$, the initial conditions, $\beta$ and $\varepsilon_{\alpha}$, provided that $\varepsilon_{\alpha}$ is sufficiently small to guarantee $1>\varepsilon_{\alpha} K_{1}(\alpha)$, in the case that $\beta=1$, and independently of $\varepsilon_{\alpha}$ if $\beta=0$. Assume that the solution $X(t)$ is not a trivial solution what is guaranteed if $\lim _{t \rightarrow \infty} e^{b t} x(t) \neq 0$, for all $b \in \mathbf{R}$. Then, it follows from (2.15) that $\lim \sup _{t_{k}-t_{0} \leq t \leq t k}\left(\ln \left|x_{t}\right|_{\alpha} / t^{\nu}\right)=$ $\mu>0$ for sufficiently large $t_{0} \in \mathbf{Z}_{0+}$, irrespective, of the $\alpha$-norm, since $\lim _{t \rightarrow \infty}\left(\ln g_{\alpha}\left(t_{0}, t\right) / t^{\nu}\right)=$ 0 for any $\alpha$-norm. By taking $\mathbf{Z}_{0+} \ni t_{0} \rightarrow \infty$, it follows that $\lim _{t \rightarrow \infty}\left(\ln \left|x_{t}\right|_{\alpha} / t^{\nu}\right)=\mu>0$. The result may be also extended to the case $\mu=0$, since then, the solution is either unbounded (for some initial conditions and multiplicity $v>1$ of the characteristic zero of $\widehat{T}^{-1}(s)$ whose real part is $\mu$ ), or it is bounded (in particular, always if $\nu=1$ for $\mu=0$ ). As a result, if $\mu \geq 0$ and there is no $b \in \mathbf{R}$ such that $e^{b t} x(t)$ converges to zero as $t \rightarrow \infty$, then

$$
\lim _{t \rightarrow \infty} \frac{\ln \left|x_{t}\right|_{\alpha}}{t^{v}}=\mu, \quad \lim _{t \rightarrow \infty} \frac{\ln \left|x_{t}\right|_{\alpha}}{t^{v+\ell}}=\mu ; \quad \forall \ell \in \mathbf{Z}_{+} \text {since } \int_{t}^{t+1} \frac{t^{\vartheta_{k}-1}}{\vartheta_{k} !} e^{\beta \sigma_{k} t} \gamma_{\alpha}(s) d s \longrightarrow 0 \text { as } t \longrightarrow \infty
$$

for all the characteristic zeros of the limiting equation (2.2) . If (2.8) holds, in particular, with $K_{0 \alpha}=0$, then the above result is also valid from (2.15) for a negative value of $\mu$. Property (ii) has been proved.

(iii) If (2.8) does not hold for $K_{0 \alpha}=0$ and all the characteristic zeros of the limiting equation (2.2) have negative real parts then it follows by using a close reasoning to that used in (ii) that the solution cannot converge asymptotically to zero but it is uniformly bounded from (2.15) since $\int_{t}^{t+1} t^{\vartheta_{k}-1} / \vartheta_{k} ! e^{\beta \sigma_{k} t} \gamma_{\alpha}(s) d s \rightarrow 0$ as $t \rightarrow \infty$ and such an integral is bounded, for all $t \in \mathbf{R}_{+}$. Thus, $\mu_{k}=0$, for all $k \geq k_{1}$ and $\mu_{k_{1}}$ is not the real part of a characteristic zero of the limiting equation (2.2) since it is not a negative real number.

The real limit $\mu_{k_{1}}$ of Theorem 2.1(ii)-(ii), provided that it exists, is called the strict Lyapunov exponent of the solution of (2.2)-(2.3) with the perturbation function 
$f\left(t, x_{t}\right)$, subject to the hypotheses of Theorem 2.1, which is the real part of an eigenvalue (or characteristic zero) of the limiting equation (2.1) if either it is positive or if it takes any arbitrary value in the case that (2.8) holds for $K_{0 \alpha}=0$ (Theorem 2.1(ii)). If all the characteristic zeros of (2.1) have negative real parts but (2.8) is not fulfilled with $K_{0 \alpha}=0$ then the strict Lyapunov exponent, if it exists, is zero so that it is not the real part of a characteristic zero of the limiting equation (2.1) (Theorem 2.1(iii)). The main extension of Theorem 2.1 for the very general functional differential equation (2.2)-(2.3) with respect to parallel previous results (see [27, Chapter IV, Theorem 5 for ordinary differential equations]; [28, Theorem 1.1, for functional differential equations] and [29]) is that the perturbation function in (2.8) is not vanishing for bounded solutions or slightly growing solutions since any bounded functions are primarily admitted as perturbations in (2.2). The extension concerning the result in [27] is restricted to the form of (2.1) which involves a wide type of delayed dynamics involving, in general, any finite numbers of point delays, finite-distributed, delays and delays generated by Volterra-type dynamics.

A notation for the subsequent lemma and theorem is the following (see [3, Chapter 7]). If $\Lambda$ is a finite set of eigenvalues of (2.1), then $P_{\Lambda}$ and $Q_{\Lambda}$ denote the generalized eigenspace associated with $\Lambda$ and the corresponding complementary subspace of $C\left(\mathbf{R}_{0+}\right)$, respectively. The phase space $C\left(\mathbf{R}_{0+}\right)$ is decomposed by $\Lambda$ into the direct $\operatorname{sum} C\left(\mathbf{R}_{0+}\right)=P_{\Lambda}\left(\mathbf{R}_{0+}\right) \oplus Q_{\Lambda}\left(\mathbf{R}_{0+}\right)$. The projections of the solution $x \in C\left(\mathbf{R}_{0+}\right)$ of (2.2), subject to (2.3), for any initial condition $\phi \in C_{e}(-h)$, onto the above subspaces are denoted by $x^{P_{\Lambda}\left(\mathbf{R}_{0^{+}}\right)}$and $x^{Q_{\Lambda}\left(\mathbf{R}_{0^{+}}\right)}$, respectively, $\forall t \in \mathbf{R}_{0+}$. Note that, although the initial conditions of (2.2)-(2.3) are in general in $C_{e}(-h)$, the corresponding unique solution of (2.2), subject to (2.3), for $t \in \mathbf{R}_{0+}$ are in $C\left(\mathbf{R}_{0+}\right)$. The whole solutions in $\mathbf{R}_{-h}$ which includes any given initial condition $\phi \in C_{e}(-h)$ then satisfying $x(t)=\phi(t), \forall t \in[-h, 0]$, and the differential equation (2.2), subject to (2.3), for $t \in \mathbf{R}_{0+}$ are in $C_{e}\left(\mathbf{R}_{-h}\right)=P_{\Lambda}\left(\mathbf{R}_{-h}\right) \oplus Q_{\Lambda e}\left(\mathbf{R}_{-h}\right)$ where $Q_{\Lambda e}\left(\mathbf{R}_{-h}\right)$ is the complementary subspace of $P_{\Lambda}\left(\mathbf{R}_{-h}\right)$ in $C_{e}\left(\mathbf{R}_{-h}\right)$. The projections of the solution onto those subspaces are $x^{P_{\Lambda}\left(\mathbf{R}_{-h}\right)}$ and $x^{Q_{\Lambda e}\left(\mathbf{R}_{-h}\right)}$, respectively, $\forall t \in \mathbf{R}_{-h}$. The following technical result follows.

Lemma 2.2. Assume that the initial condition of (2.2)-(2.3) is $x(t)=\phi(t), \forall t \in[-h, 0]$ for any given $\phi \in C_{e}(-h)$. The unique solution of (2.2), subject to (2.3) on $\mathbf{R}_{0+}$, and identified with $\phi(t) \forall t \in$ $[-h, 0]$, satisfies with unique decompositions:

$$
\begin{gathered}
x_{t+h}=x_{t+h}^{P_{\Lambda}\left(\mathbf{R}_{0+}\right)}+x_{t+h}^{Q_{\Lambda}\left(\mathbf{R}_{0+}\right)} ; \quad \forall t \in \mathbf{R}_{0+}, \\
x_{t}=x_{t}^{P_{\Lambda}\left(\mathbf{R}_{-h}\right)}+x_{t}^{Q_{\Lambda e}\left(\mathbf{R}_{-h}\right)} ; \quad \forall t \in \mathbf{R}_{0+}, \\
x_{0}=\phi_{0}^{P_{\Lambda}\left(\mathbf{R}_{-h}\right)}=\phi_{0}^{P_{\Lambda}\left(\mathbf{R}_{0+}\right)}=\phi_{0}^{P_{\Lambda}\left(\mathbf{R}_{0+}\right)}=x_{0}^{P_{\Lambda}\left(\mathbf{R}_{-h}\right)}+x_{0}^{Q_{\Lambda e}\left(\mathbf{R}_{-h}\right)}=\phi_{0}^{P_{\Lambda}\left(\mathbf{R}_{-h}\right)}+\phi_{0}^{Q_{\Lambda e}\left(\mathbf{R}_{-h}\right)} .
\end{gathered}
$$

Proof. The first relation follows from $C\left(\mathbf{R}_{0+}\right)=P_{\Lambda}\left(\mathbf{R}_{0+}\right) \oplus Q_{\Lambda}\left(\mathbf{R}_{0_{+}}\right)$, and the superposition principle for linear systems building the solution for $t(\geq h) \in \mathbf{R}_{0+}$ by projecting the function of initial conditions into the complementary subspaces $P_{\Lambda}\left(\mathbf{R}_{0+}\right)$ and $Q_{\Lambda}\left(\mathbf{R}_{0+}\right)$ in $C\left(\mathbf{R}_{0+}\right)$ subject to the constraint $x(t)=\phi(t)$, for all $t \in[-h, 0]$. The second relation follows from $C_{e}\left(\mathbf{R}_{-h}\right)=$ $P_{\Lambda}\left(\mathbf{R}_{-h}\right) \oplus Q_{\Lambda e}\left(\mathbf{R}_{-h}\right)$ again from the superposition principle with $x(t)=\phi(t)$, for all $t \in[-h, 0]$. The third relation follows from $x_{0}=x(0)=\phi(0)$ and the superposition principle applied to the solution at $t=0$.

The intuitive meaning of Lemma 2.2 is that for $t \geq 0, x(t)$ is decomposed uniquely as a sum of a function in $P_{\Lambda}\left(\mathbf{R}_{0+}\right)$ and another one in its complementary in $C\left(\mathbf{R}_{0+}\right)$, even for initial conditions in $C_{e}(-h)$, rather than in the more restrictive set $C(-h)$. However, the 
complementary set $Q_{\Lambda e}\left(\mathbf{R}_{-h}\right)$ of $P_{\Lambda}\left(\mathbf{R}_{-h}\right)$ in $C\left(\mathbf{R}_{-h}\right)$ replaces $Q_{\Lambda}\left(\mathbf{R}_{0+}\right) t \leq 0$, since $x_{0}=\phi_{0}$ for any given $\phi \in C_{e}(-h)$. Note that $x_{t}^{\left(\mathbf{R}_{-h}\right)}=\phi_{t}^{P_{\Lambda}\left(\mathbf{R}_{-h}\right)}+\phi_{t}^{Q_{\Lambda e}\left(\mathbf{R}_{-h}\right)}, \forall t \in \mathbf{R}_{0+}$ is untrue except for $\phi \in C(-h)$.

Theorem 2.3. Let $x$ be a solution of (2.2)-(2.3) satisfying the hypotheses of Theorem 2.1 with a finite strict Lyapunov exponent $\mu(x)=\mu_{k_{1}}=\mu$. Consider generalized eigenspaces $P_{0}\left(\mathbf{R}_{0+}\right)=P_{\Lambda_{0}}\left(\mathbf{R}_{0_{+}}\right)$, $P_{1}\left(\mathbf{R}_{0+}\right)=P_{\Lambda_{1}}\left(\mathbf{R}_{0+}\right)$ and $Q\left(\mathbf{R}_{0+}\right)=Q_{\Lambda}\left(\mathbf{R}_{0+}\right)$ for $t \in \mathbf{R}_{0+}$ and, also, generalized eigenspaces $P_{0}\left(\mathbf{R}_{-h}\right)=$ $P_{\Lambda_{0}}\left(\mathbf{R}_{-h}\right), P_{1}\left(\mathbf{R}_{-h}\right)=P_{\Lambda_{1}}\left(\mathbf{R}_{-h}\right)$ and $Q_{e}\left(\mathbf{R}_{-h}\right)=Q_{\Lambda_{e}}\left(\mathbf{R}_{-h}\right)$ for $t \in \mathbf{R}_{-h}$, where the spectral sets $\Lambda_{0}, \Lambda_{1}$ and $\Lambda$ each generating the two corresponding eigenspaces, are defined by,

$$
\begin{gathered}
\Lambda_{0}=\Lambda_{0}(\mu):=\left\{\lambda: \operatorname{det} \widehat{T}^{-1}(\lambda)=0, \operatorname{Re} \lambda=\mu\right\}, \\
\Lambda_{1}=\Lambda_{1}(\mu):=\left\{\lambda: \operatorname{det} \widehat{T}^{-1}(\lambda)=0, \operatorname{Re} \lambda>\mu\right\}, \\
\Lambda=\Lambda_{0} \cup \Lambda_{1}=\Lambda(\mu):=\left\{\lambda: \operatorname{det} \widehat{T}^{-1}(\lambda)=0, \operatorname{Re} \lambda \geq \mu\right\} .
\end{gathered}
$$

Then, the following properties hold,

(i)

(i.1) $\Lambda_{0} \neq \varnothing, \Lambda \neq \Phi$ if $\mu \in \mathbf{R}_{0+}$,

(i.2) $\Lambda_{0} \neq \varnothing$ if $\mu \in \mathbf{R}$ and, furthermore, (2.8) holds with $K_{0 \alpha}=0$,

(i.3) $\Lambda=\Lambda_{0}=\Lambda_{1}=\varnothing$ if all the eigenvalues of (2.1) have negative real parts and, furthermore, (2.8) does not hold with $K_{0 \alpha}=0$,

(i.4) $\Lambda_{1}=\varnothing$ if any of the following conditions hold:

(1) No eigenvalue of (2.1) is in $\mathbf{R}_{0+}$,

(2) No eigenvalue of (2.1) is in $\mathbf{R}_{+}$and, furthermore, (2.8) does not hold with $K_{0 \alpha}=0$,

(3) (2.8) holds with $K_{0 \alpha}=0$.

(ii) The solution of (2.2) under arbitrary initial conditions $\phi \in C_{e}(-h)$, subject to a perturbation function (2.3), satisfies,

$$
\begin{gathered}
x_{t+h}=x_{t+h}^{P_{0}\left(\mathbf{R}_{0+}\right)}+x_{t+h}^{P_{1}\left(\mathbf{R}_{0+}\right)}+x_{t+h}^{Q\left(\mathbf{R}_{0+}\right)} ; \quad \forall t \in \mathbf{R}_{0+,} \\
x_{t}=x_{t}^{P_{0}\left(\mathbf{R}_{-h}\right)}+x_{t}^{P_{1}\left(\mathbf{R}_{-h}\right)}+x_{t}^{Q_{e}\left(\mathbf{R}_{-h}\right)} ; \quad \forall t \in \mathbf{R}_{0+}, \\
x_{0}=\phi_{0}^{P_{0}\left(\mathbf{R}_{-h}\right)}=x_{0}^{P_{0}\left(\mathbf{R}_{0+}\right)}=\phi_{0}^{P_{0}\left(\mathbf{R}_{0+}\right)}=x_{0}^{P_{0}\left(\mathbf{R}_{-h}\right)}+x_{0}^{P_{1}\left(\mathbf{R}_{-h}\right)}+x_{0}^{Q_{e}\left(\mathbf{R}_{-h}\right)}=\phi_{0}^{P_{0}\left(\mathbf{R}_{-h}\right)}+x_{0}^{P_{1}\left(\mathbf{R}_{-h}\right)}+\phi_{0}^{Q_{e}\left(\mathbf{R}_{-h}\right)} .
\end{gathered}
$$

(iii) The solution of (2.2) under arbitrary initial condition $\phi \in C_{e}(-h)$, subject to a perturbation function (2.3), satisfies,

$$
\begin{array}{lll}
x_{t+h}^{P_{1}\left(\mathbf{R}_{0+}\right)}=O\left(\left|x_{t+h}^{P_{0}\left(\mathbf{R}_{0+}\right)}\right|\right), & x_{t+h}^{Q\left(\mathbf{R}_{0+}\right)}=O\left(\left|x_{t+h}^{P_{0}\left(\mathbf{R}_{0+}\right)}\right|\right), & x_{t+h}=O\left(\left|x_{t+h}^{P_{0}\left(\mathbf{R}_{0+}\right)}\right|\right) ; \quad \forall t \in \mathbf{R}_{0+} \\
x_{t}^{P_{1}\left(\mathbf{R}_{-h}\right)}=O\left(\left|x_{t}^{P_{0}\left(\mathbf{R}_{-h}\right)}\right|\right), & x_{t}^{Q_{e}\left(\mathbf{R}_{-h}\right)}=O\left(\left|x_{t}^{P_{0}\left(\mathbf{R}_{-h}\right)}\right|\right), & x_{t}=O\left(\left|x_{t}^{P_{0}\left(\mathbf{R}_{-h}\right)}\right|\right) ; \quad \forall t \in \mathbf{R}_{0+}
\end{array}
$$


Furthermore, if (2.8) holds with $K_{0 \alpha}=0$ then, as $t \rightarrow \infty$ :

$$
\begin{array}{lll}
x_{t+h}^{P_{1}\left(\mathbf{R}_{0+}\right)}=o\left(\left|x_{t+h}^{P_{0}\left(\mathbf{R}_{0+}\right)}\right|\right), & x_{t+h}^{Q\left(\mathbf{R}_{0+}\right)}=o\left(\left|x_{t+h}^{P_{0}\left(\mathbf{R}_{0+}\right)}\right|\right), \quad x_{t+h}=o\left(\left|x_{t+h}^{P_{0}\left(\mathbf{R}_{0+}\right)}\right|\right) ; \quad \forall t \in \mathbf{R} 0+ \\
x_{t}^{P_{1}\left(\mathbf{R}_{-h}\right)}=o\left(\left|x_{t}^{P_{0}\left(\mathbf{R}_{-h}\right)}\right|\right), & x_{t}^{Q_{e}\left(\mathbf{R}_{-h}\right)}=o\left(\left|x_{t}^{P_{0}\left(\mathbf{R}_{-h}\right)}\right|\right), \quad x_{t}=o\left(\left|x_{t}^{P_{0}\left(\mathbf{R}_{-h}\right)}\right|\right) ; \quad \forall t \in \mathbf{R} 0+
\end{array}
$$

(iv) The solution of the limiting equation (2.1) satisfies (2.23) as $t \rightarrow \infty$.

(v) The solution of (2.2), under arbitrary initial conditions $\phi \in C_{e}(-h)$ and subject to a perturbation function (2.3), satisfies:

$$
\begin{gathered}
x_{t+h}=x_{t+h}^{P_{0}}+x_{t+h}^{P_{1}}+x_{t+h}^{Q} ; \quad \forall t \in \mathbf{R}_{0+} \\
x_{t}=x_{t}^{P_{0}}+x_{t}^{P_{1}}+x_{t}^{Q_{e}} ; \quad \forall t \in \mathbf{R}_{0+}
\end{gathered}
$$

which is identical to

$$
x_{t}=x_{t}^{P_{0}}+x_{t}^{P_{1}}+x_{t}^{Q} ; \quad \forall t \in \mathbf{R}_{-h}
$$

under the restriction $\phi \in C(-h)$ for the initial conditions with $x(t)=\phi(t)$. Also,

$$
x_{t}^{P_{1}}=O\left(\left|x_{t}^{P_{0}}\right|\right), \quad x_{t}^{Q}=O\left(\left|x_{t}^{P_{0}}\right|\right), \quad x_{t}=O\left(\left|x_{t}^{P_{0}}\right|\right) ; \quad \forall t \in \mathbf{R}_{0+}, \forall \phi \in C_{e}(-h) .
$$

If (2.8) holds with $K_{0 \alpha}=0$ then, as $t \rightarrow \infty$ :

$$
x_{t+h}^{P_{1}}=o\left(\left|x_{t}^{P_{0}}\right|\right), \quad x_{t+h}^{Q}=o\left(\left|x_{t+h}^{P_{0}}\right|\right), \quad x_{t}=o\left(\left|x_{t+h}^{P_{0}}\right|\right) ; \quad \forall t \in \mathbf{R}_{0+},
$$

which leads to

$$
x_{t}^{P_{1}}=o\left(\left|x_{t}^{P_{0}}\right|\right), \quad x_{t}^{Q}=o\left(\left|x_{t}^{P_{0}}\right|\right), \quad x_{t}=o\left(\left|x_{t}^{P_{0}}\right|\right) ; \quad \forall t \in \mathbf{R}_{0+}
$$

under the restriction $\phi \in C(-h)$ for the initial conditions with $x(t)=\phi(t)$. The solution of the limiting equation (2.1) satisfies (2.27)-(2.28) as $t \rightarrow \infty$.

Proof. Properties (i) are direct consequences of Theorem 2.1 [(ii)-(iii)] as follows. property (i.1) follows by noting that $\operatorname{det} \widehat{T}^{-1}(\lambda)=0$ for some $\lambda \in \mathrm{C}$ implies that $\lambda$ is a characteristic zero of (2.1) of nonnegative real part from (2.7). Assume that $\mu \in \mathbf{R}_{0+} \wedge \Lambda_{0}=\varnothing \Leftrightarrow \hat{\lambda}:=\max (\operatorname{Re}(\lambda)$ : $\lambda \in C Z) \neq \mu \geq 0$ where $C Z \subset \mathrm{C}$ is the set of characteristic zeros of the limiting equation (2.1). Thus, if $\hat{\lambda} \neq \mu=0$, then the current equation (2.2)-(2.3) is bounded while the limiting one (2.1) is either globally asymptotically stable or unstable so that they cannot converge asymptotically to each other which is a contradiction so that $\Lambda \supset \Lambda_{0} \neq \varnothing$. If $\hat{\lambda} \neq \mu>0$ then the current equation is unstable which implies that the limiting one should satisfy $0<\mu \neq \hat{\lambda}>0$ 
to be also unstable but the asymptotic convergence of their respective solutions to each other is only possible if $\hat{\imath}=\mu>0$. Thus, again $\Lambda \supset \Lambda_{0} \neq \varnothing$ and property (i.1) has been proven. On the other hand, $K_{0 \alpha}=0 \Rightarrow \hat{\lambda}=\mu \Rightarrow \Lambda_{0} \neq \varnothing$ and property (i.2) is proven. Also, $\Lambda=\Lambda_{0}=\Lambda_{1}=\varnothing \Rightarrow \bar{\lambda}<\mu$. Since, in addition, (2.2)-(2.3) is the limiting equation of (2.1) then $\bar{\lambda}<\mu<0$ and $K_{0 \alpha}>0$. Therefore, $K_{0 \alpha}=0$ and property (i.3) have been proven. Property (i.4) follows from:

$$
\Lambda_{1}=\varnothing \Longrightarrow \hat{\lambda} \leq \mu<0 \vee\left(\widehat{\lambda} \leq \mu=0 \wedge K_{0 \alpha}=0\right) \vee\left(0<\widehat{\lambda} \leq \mu>0 \wedge K_{0 \alpha}=0\right)
$$

in order to the solutions of (2.2)-(2.3) and (2.1) to asymptotically to converge to each other. Property (i) has been fully proven.

Property (ii) is a direct consequence of Lemma 2.2 since $\Lambda_{0}$ and $\Lambda_{1}$ are disjoint sets which implies that

$$
\begin{gathered}
C\left(\mathbf{R}_{0+}\right)=P_{0}\left(\mathbf{R}_{0+}\right) \oplus P_{1}\left(\mathbf{R}_{0+}\right) \oplus Q_{\Lambda}\left(\mathbf{R}_{0+}\right), \\
C_{e}\left(\mathbf{R}_{-h}\right)=P_{0}\left(\mathbf{R}_{-h}\right) \oplus P_{1}\left(\mathbf{R}_{-h}\right) \oplus Q_{\Lambda e}\left(\mathbf{R}_{-h}\right) .
\end{gathered}
$$

Property (iii) is directly proven as follows. Equations (2.22) are a direct consequence of property (ii). On the other hand, (2.23) are a direct consequence of (2.22) if (2.8) holds for $K_{0 \alpha}=0$ so that property (iii) follows. in (2.3).

Property (iv) follows from property (iii) as particular case for $f\left(t, x_{t}\right)=0 ; \forall t \in \mathbf{R}_{-h}$

Property (v) is a direct consequence of Properties (i)-(iv) In particular, the relative growing properties of "O" $\mathrm{O}$-type of the various parts of the solution of (2.2)-(2.3) are embedded from property (iii) into similar properties for the solution strings of length $h$. The part of property ( $\mathrm{v}$ ) concerning the relative growing properties of " $\mathrm{o}$ "-type of the various parts of the solution of (2.2)-(2.3) and that concerning the limiting equation follows directly under a close reasoning.

Note that in Theorem 2.3, the various results obtained for "Landau small-o" notation, referred to limits as $t \rightarrow \infty$ imply, as usual, that parallel results for "Landau big-O" notation stand for all $t \in \mathbf{R}_{0+}$ but the converse is not true. The results concerning "Landau big$\mathrm{O}^{\prime \prime}$ notation in Theorem 2.3(iii) for the perturbed functional equation (2.2)-(2.3) are new for the studied class of functional equations, related to the background literature, since the perturbation function is allowed to take bounded nonzero values even if the limiting equation is globally asymptotically stable and it is not requested to grow asymptotically at most linearly with $x_{t}$. The results concerning "Landau big-O" notation imply that the solution of the perturbed functional equation is uniformly bounded for any bounded function of initial conditions of the given class for all time so that the functional differential equation is globally uniformly Lyapunov stable provided that the perturbation (2.3) satisfies the given hypotheses. A technical result concerning the boundedness of the evolution operator, which will be then useful to derive further results, and stability properties of the differential systems (2.1) and (2.2)-(2.3) follows. 
Theorem 2.4. The following properties hold:

(i) The evolution operator of the limiting functional differential equation (2.1) satisfies the subsequent relations.

$$
\begin{aligned}
& \|T(t, 0)\|_{\alpha} \leq K_{1}(\alpha)\left\|I_{n}\right\|_{\alpha} \max \left(1, \frac{t^{v-1}}{v !} e^{\mu t}\right) ; \quad \forall t \in \mathbf{R}_{0+} \\
& |T(t, 0)|_{\alpha} \leq K_{1}(\alpha)\left\|I_{n}\right\|_{\alpha} \max \left(1, \frac{t^{v-1}}{v !} e^{\mu t} \max \left(1, \sum_{i=0}^{v-2} t^{1-v+i} h^{v-1-i} e^{\mu h}\right)\right) ; \quad \forall t \in \mathbf{R} 0+ \\
& \|\dot{T}(t, 0)\|_{\alpha} K_{2}(\alpha) \sup _{0 \leq \tau \leq t}\left|T_{\tau}^{C\left(\mathbf{R}_{0+}\right)}\right|_{\alpha} ; \quad \forall t \in \mathbf{R}_{0+} \\
& |T(t, 0)|_{\alpha} \leq K_{2}(\alpha) \sup _{0 \leq \tau \leq t}\left|T_{\tau}^{C\left(\mathbf{R}_{0+}\right)}\right|_{\alpha} \\
& \quad \leq K_{1}(\alpha) K_{2}(\alpha)\left\|I_{n}\right\|_{\alpha} \max \left(1, \frac{t^{\nu-1}}{v !} e^{\mu t} \max \left(1, \sum_{i=0}^{v-2} t^{1-v+i} h^{\nu-1-i} e^{\mu h}\right)\right) ; \quad \forall t \in \mathbf{R}_{0+}
\end{aligned}
$$

Proof. (i) The evolution operator satisfies the limiting functional differential equation (2.1):

$$
\dot{T}(t, 0)=\sum_{i=0}^{m} A_{i} T\left(t-h_{i}, 0\right)+\sum_{i=0}^{m^{\prime}} \int_{0}^{t} d \alpha_{i}(\tau) A_{\alpha_{i}} T\left(t-h_{i}^{\prime}, \tau\right)+\sum_{i=m+l}^{m^{\prime}+m^{\prime \prime}} \int_{h_{i}^{\prime}}^{0} d \alpha_{i}(\tau) A_{\alpha_{i}} T(t, \tau)
$$

for $t \in \mathbf{R}_{0+}$ subject to initial conditions $T(0,0)=I_{n}$ (i.e., the $n$th identity matrix) and $T(t, 0)=0, t \in[-h, 0)$. Thus, it satisfies also the unforced (2.2) (i.e., for $\gamma_{\alpha}=|\phi|_{\alpha}=K_{0 \alpha}=0$ ). This leads directly to (2.31). However, (2.32) follows by using the Newton binomial to expand $\left((t+h)^{v-1} / v !\right) e^{\mu(t+h)}$ and the fact that the maximum of the real exponential function within the real interval $[0, t]$ is reached at the boundary. Equation (2.33) follows by the inspection of (2.36) for some norm-dependent $K_{2}(\alpha) \in \mathbf{R}_{+}$which depends on the various matrices of parameters of the limiting functional differential equation (2.1). Equation (2.34) follows from (2.33) and (2.36). Finally, (2.35) follows from (2.34) and (2.32). Property (i) has been proved.

(ii) For sufficiently small constant $\left(\beta \varepsilon_{\alpha}\right)$, the evolution operator as a function of time is of exponential order whose norm time-function satisfies:

$$
\begin{aligned}
\|T(t, 0)\|_{\alpha} & =\sup _{t_{k} t_{0} \leq t \leq t_{k}}\left(\left|x_{t}\right|_{\alpha}\right) \\
& \leq\left(1-\beta \varepsilon_{\alpha} K_{1}(\alpha)\right)^{-1}\left(K_{1}(\alpha) \frac{\left(t_{k}-t_{0}\right)^{\nu 1}}{v !}\left[e^{\mu\left(t_{k}-t_{0}\right)}\left\|I_{n}\right\|_{\alpha}\right]\right),
\end{aligned}
$$


which converges exponentially to zero as $t \rightarrow \infty$ if the strict Lyapunov exponent $\mu$ is negative. In this case, the limiting differential functional equation is globally uniformly exponentially Lyapunov stable whose solution satisfies asymptotically:

$$
\begin{aligned}
\left\|x\left(t_{k}\right)\right\|_{\alpha}= & \sup _{t_{k}-t_{0} \leq t \leq t_{k}}\left(\left|x_{t}\right|_{\alpha}\right) \\
\leq & \left(1-\beta \varepsilon_{\alpha} K_{1}(\alpha)\right)^{1} \\
& \times\left(K_{1}(\alpha) \frac{\left(t_{k}-t_{0}\right)^{\nu-1}}{v !}\left[e^{\mu\left(t_{k}-t_{0}\right)}\left\|x\left(t_{0}^{+}\right)\right\|_{\alpha}+\left|\frac{e^{\mu\left(t_{k} t_{0}\right)}\left(1-e^{\mu h}\right)}{\mu}\right|\left(|\phi|_{\alpha}\right)\right]\right),
\end{aligned}
$$

so that it converges exponentially to zero as $t \rightarrow \infty$ for any admissible function of initial conditions. The differential equation (2.2), subject to (2.3) is globally uniformly Lyapunov stable if $\mu \leq 0$ and its solution satisfies:

$$
\begin{aligned}
\left\|x\left(t_{k}\right)\right\|_{\alpha}= & \sup _{t_{k}-t_{0} \leq t \leq t_{k}}\left(\left|x_{t}\right|_{\alpha}\right) \\
\leq & \left(1-\beta \varepsilon_{\alpha} K_{1}(\alpha)\right)^{-1} \\
& \times\left(K_{1}(\alpha) \frac{\left(t_{k}-t_{0}\right)^{\nu-1}}{v !}\left[e^{\mu\left(t_{k}-t_{0}\right)}\left\|x\left(t_{0}^{+}\right)\right\|_{\alpha}+\left|\frac{e^{\mu\left(t_{k}-t_{0}\right)}\left(1-e^{\mu h}\right)}{\mu}\right|\left(|\phi|_{\alpha}+K_{0 \alpha}\right)\right]\right),
\end{aligned}
$$

for large $t$ and converges exponentially to zero (i.e., it is globally uniformly exponentially Lyapunov stable) if $\mu<0$ and the perturbation function has an upper-bounding function with $K_{0 \alpha}=0$.

(ii) It follows directly from (2.12).

The evolution operator $T: \mathbf{R}_{0+} \times \mathbf{C}^{n} \rightarrow \mathbf{C}^{n}$ explicits the solutions of the limiting equation (2.5) and the perturbed one (2.6) for each function of initial conditions. Then, let $\left(T_{S}(t)\right)_{t \in \mathbf{R}_{0+}}$ be the solution semigroup of the linear autonomous equation (2.1), which is unique for $t \in \mathbf{R}_{0+}$ for each $\phi \in C_{e}(-h)$ and whose infinitesimal generator is $A$ satisfying $\dot{\varphi}=A \varphi, \forall \varphi \in \operatorname{Dom}(A):=\left\{\varphi \in C_{e}\left(\mathbf{R}_{-h}\right): \exists \dot{\varphi} \in C\left(\mathbf{R}_{0+}\right), \forall t \in \mathbf{R}_{0+} \wedge \dot{\varphi}(0)=L \varphi(0)\right\}$. Thus, the string $x_{t}(\phi)=\left(T_{s} \phi\right)(t)$ of the solution of the limiting functional differential equation (2.1) within $[t-h, t]$ is defined from (2.5) as follows:

$$
\begin{gathered}
x_{t}(\phi)=\left(T_{s} \phi\right)(t):=T(t-\theta, 0) x\left(0^{+}\right)+\int_{-h}^{0} T(t-\theta, \tau) \phi(\tau) U(\tau) d \tau, \quad \forall \theta \in[0, \min (t, h)], \\
\left.x_{t}(\phi)=\left(T_{s} \phi\right)(t)=0, \quad \forall \theta \in\right] 0, \min (t, h)\left[:=\overline{[0, \min (t, h)]} \cap \mathbf{R}_{0+}, \quad \forall t \in \mathbf{R}_{0+} ;\right.
\end{gathered}
$$


and the corresponding solution string of the perturbed functional differential equation (2.2)(2.3) is then defined follows:

$$
\begin{aligned}
x_{t}(\phi)= & \left(T_{s} \phi\right)(t) \\
:= & T(t-\theta, 0) x\left(0^{+}\right)+\int_{-h}^{0} T(t-\theta, \tau) \phi(\tau) U(\tau) d \tau \\
& +\int_{0}^{t} T(t-\theta, \tau) f\left(\tau, x_{\tau}\right) d \tau, \quad \forall \theta \in[0, \min (t, h)], \\
x_{t}(\phi)= & \left(T_{s} \phi\right)(t)=0, \quad \forall \theta \in \overline{[0, \min (t, h)]} \cap \mathbf{R} ; \forall t \in \mathbf{R}_{0+} .
\end{aligned}
$$

The transposed equation associated with (2.1) is

$$
\begin{aligned}
\dot{y}(t) & =\mathrm{L}^{*} y_{t} \\
& :=\sum_{i=0}^{m} y\left(t-h_{i}\right) A_{i}^{*}+\sum_{i=0}^{m^{\prime}} \int_{0}^{t} y\left(t-\tau-h_{i}^{\prime}\right) A_{\alpha_{i}}^{*} d \alpha_{i}^{*}(\tau)+\sum_{i=m^{\prime}+1}^{m^{\prime}+m^{\prime \prime}} \int_{t-h_{i}^{\prime}}^{t} y(\tau) A_{\alpha_{i}}^{*} d \alpha_{i}^{*}(\tau),
\end{aligned}
$$

where the superscript $*$ denotes the adjoint operators of the corresponding un-superscripted ones. In particular, for matrices, it denotes the conjugate transposes of the corresponding unsuperscripted ones. Thus, $y(t)$ is a $n$-dimensional complex row vector. The phase space for (2.42) on $\mathbf{R}_{0+}$ is $C^{\prime}\left(\mathbf{R}_{0+}\right):=C\left(\mathbf{R}_{0+}, \mathbf{C}^{n *}\right)$. Corresponding spaces of functions taking into account the more general spaces for initial conditions are $C^{\prime}(h):=C\left([0, h], \mathbf{C}^{n^{*}}\right), C_{e}^{\prime}(h):=C\left([0, h], \mathbf{C}^{n^{*}}\right)$ and $C^{\prime}\left(\mathbf{R}_{-h}\right):=C\left(\mathbf{R}_{-h}, C^{n^{*}}\right)$. Let $\Lambda$ be a finite set of eigenvalues of (2.1) and let $\Phi_{\Lambda}$ be a basis for the generalized eigenspace $P_{\Lambda},[27,28]$. Then, there exists a square $n$-matrix $B_{\Lambda}$, with $\operatorname{sp}\left(B_{\Lambda}\right)=\operatorname{sp}(\Lambda)$, such that the subsequent relations hold:

$$
A \Phi_{\Lambda}=\Phi_{\Lambda} B_{\Lambda}, \quad \Phi_{\Lambda}(\tau)=\Phi_{\Lambda}(0) e^{B_{\Lambda} \tau} \quad(\forall \tau \in[-h, 0]), \quad T(t, 0) \Phi_{\Lambda}=\Phi_{\Lambda} e^{B_{\Lambda} t}
$$

The relations (2.43) yield via direct computation property (i) of the subsequent result since $B_{\Lambda}$ commutes with $e^{B_{\Lambda} t}$. Property (ii) is a direct consequence of (2.36) subject to $T(0,0)=I_{n}$ and $T(t, 0)=0$ for $t<0$.

Proposition 2.5. The two following properties hold.

(i) The following relations hold, for all $t \in \mathbf{R}_{0+}$ :

$$
\begin{aligned}
T(t, 0) \Phi_{\Lambda} B_{\Lambda} & =\Phi_{\Lambda} e^{B_{\Lambda} t} B_{\Lambda}=\Phi_{\Lambda} B_{\Lambda} e^{B_{\Lambda} t}=A \Phi_{\Lambda} e^{B_{\Lambda} t}=A T(t, 0) \Phi_{\Lambda} \\
\dot{T}(t, 0) \Phi_{\Lambda} B_{\Lambda} & =\Phi_{\Lambda} B_{\Lambda}^{2} e^{B_{\Lambda} t}=A \Phi_{\Lambda} B_{\Lambda} e^{B_{\Lambda} t}=A^{2} \Phi_{\Lambda} e^{B_{\Lambda} t}=A \Phi_{\Lambda} e^{B_{\Lambda} t} B_{\Lambda} \\
& =\Phi_{\Lambda} B_{\Lambda} e^{B_{\Lambda} t} B_{\Lambda}=T(t, 0) \Phi_{\Lambda} B_{\Lambda}^{2}=T(t, 0) A \Phi_{\Lambda} B_{\Lambda}=A^{2} T(t, 0) \Phi_{\Lambda} \\
& =A T(t, 0) \Phi_{\Lambda} B_{\Lambda}=A T(t, 0) A \Phi_{\Lambda} .
\end{aligned}
$$


(ii) The evolution operator of the solution of (2.1) is uniquely given by

$$
\begin{aligned}
T(t, 0)=e^{A_{0} t}\left(I_{n}+\left[\int_{0}^{t} e^{-A_{0} \tau}(\right.\right. & \sum_{i=1}^{m} A_{i} T\left(t-h_{i}, \tau\right) \\
& +\sum_{i=0}^{m^{\prime}} \int_{0}^{\tau} d \alpha_{i}(\theta) A_{\alpha_{i}} T\left(\tau-h_{i}^{\prime}, \theta\right) \\
& \left.\left.\left.+\sum_{i=m^{\prime}+1}^{m^{\prime}+m^{\prime \prime}} \int_{h_{i}^{\prime}}^{0} d \alpha_{i}(\theta) A_{\alpha_{i}} T(\tau, \theta)\right) d \tau\right]\right)
\end{aligned}
$$

for all $t \in \mathbf{R}_{0+}$ with $T(0,0)=I_{n}$ and $T(t, 0)=0$ for $t \in[-h, 0]$.

Equations (2.43)-(2.45) are useful for the asymptotic analysis of comparison of the solutions of (2.2)-(2.3) with that of its limiting equation obtained from (2.1) which follows. The solutions of $P_{\Lambda}$ can be extended to $\forall t \in \mathbf{R}$ by $T(t, 0) \Phi_{\Lambda} a=\Phi_{\Lambda} e^{B_{\Lambda} t} a$, where $a$ is of dimension compatible with the order of $\Phi_{\Lambda}$. Let $Q_{\Lambda}$ be the complementary eigenspace to $P_{\Lambda}$. Now, use appropriate notations for the corresponding subspaces on $\mathbf{R}_{0+}$ and their extensions to $\mathbf{R}_{-h}$ to consider more general initial conditions (on $C_{e}(-h)$ ) for (2.1) and (2.2)(2.3) than bounded continuous functions in a Banach space leading to the uniquely defined decompositions $C_{e}\left(\mathbf{R}_{-h}\right)=P_{\Lambda}\left(\mathbf{R}_{-h}\right) \oplus Q_{\Lambda e}\left(\mathbf{R}_{-h}\right)$ and $C\left(\mathbf{R}_{0+}\right)=P_{\Lambda}\left(\mathbf{R}_{0+}\right) \oplus Q_{\Lambda}\left(\mathbf{R}_{0_{+}}\right)$. Then, given a function of initial conditions $\phi \in C_{e}(-h)$ the decomposition $\phi_{0}=\left.\phi_{t}\right|_{t=0}=\phi_{0}^{P_{\Lambda}\left(\mathbf{R}_{-h}\right)}+\phi_{0}^{Q_{\Lambda}\left(\mathbf{R}_{-h}\right)}$ is unique. Also, the unique solution of (2.1) and that of (2.2), subject to (2.3), are uniquely decomposable in $\mathbf{R}_{0+}$ as

$$
\begin{gathered}
x_{t+h}=x_{t+h}^{P_{\Lambda}\left(\mathbf{R}_{0+}\right)}+x_{t+h}^{Q_{\Lambda}\left(\mathbf{R}_{0+}\right)} \\
x_{t+h}^{P_{\Lambda}\left(\mathbf{R}_{0+}\right)}=\Phi_{\Lambda}\left(\Psi_{\Lambda}, x_{t+h}\right) \in P_{\Lambda}\left(\mathbf{R}_{0+}\right), \quad x_{t+h}^{Q_{\Lambda}\left(\mathbf{R}_{0+}\right)}=x_{t+h}-x_{t+h}^{P_{\Lambda}\left(\mathbf{R}_{0+}\right)} \in Q_{\Lambda}\left(\mathbf{R}_{0+}\right) \quad \forall t \in \mathbf{R}_{0+}
\end{gathered}
$$

via the direct sum of subspaces $C\left(\mathbf{R}_{0+}\right)=P_{\Lambda}\left(\mathbf{R}_{0+}\right) \oplus Q_{\Lambda}\left(\mathbf{R}_{0+}\right)$. The solution iincluding initial conditions defined by $x(t)=\phi(t)$ for $t \in[-h, 0]$ is uniquely decomposable in $\mathbf{R}_{-h}$ as

$$
\begin{gathered}
x_{t}=x_{t}^{P_{\Lambda}\left(\mathbf{R}_{-h}\right)}+x_{t}^{Q_{\Lambda e}\left(\mathbf{R}_{-h}\right)}, \\
x_{t}^{P_{\Lambda}\left(\mathbf{R}_{-h}\right)}=\Phi_{\Lambda}\left(\Psi_{\Lambda}, x_{t}\right) \in P_{\Lambda}\left(\mathbf{R}_{-h}\right), \quad x_{t}^{Q_{\Lambda e}\left(\mathbf{R}_{-h}\right)}=x_{t}-x_{t}^{P_{\Lambda}\left(\mathbf{R}_{-h}\right)} \in Q_{\Lambda e}\left(\mathbf{R}_{-h}\right) \quad \forall t \in \mathbf{R}_{0+}
\end{gathered}
$$

via the direct sum of subspaces $C_{e}\left(\mathbf{R}_{-h}\right)=P_{\Lambda}\left(\mathbf{R}_{-h}\right) \oplus Q_{\Lambda e}\left(\mathbf{R}_{-h}\right)$. 


\section{Asymptotic Behavior and Asymptotic Comparison}

The string solution (2.6) of (2.2)-(2.3) for $\theta \in[t-h, t]$, point-wise defined by $x(t)=\phi(t)$, $t \in[-h, 0]$, any given $\phi \in C_{e}(-h)$, and

$$
\begin{array}{r}
x(t+\theta)=T(t+\theta, 0) x\left(0^{+}\right)+\int_{-h}^{0} T(t+\theta, \tau) \phi(\tau) U(\tau) d \tau+\int_{0}^{t+\theta} T(t+\theta, \tau) f\left(\tau, x_{\tau}\right) d \tau, \\
\forall \theta \in[-h, 0], t \geq h,
\end{array}
$$

may be expressed equivalently via the solution semigroup of the limiting equation (2.1) as

$$
\begin{aligned}
x_{t}(\phi, \theta) & =x_{t}^{*}(\phi, \theta)+\int_{0}^{t+\theta} d[K(t+\theta, \tau)] f\left(\tau, x_{\tau}\right) \\
& =\left(T_{s}(t, 0) \phi\right)(\theta)+\int_{0}^{t} d[K(t+\theta, \tau)] f\left(\tau, x_{\tau}\right) \\
& =T(t+\theta, 0) \phi\left(0^{+}\right)+\int_{-h}^{0} T(t+\theta, \tau) \phi(\tau) U(\tau) d \tau+\int_{0}^{t+\theta} T(t+\theta, \tau) f\left(\tau, x_{\tau}\right) d \tau,
\end{aligned}
$$

where $x_{(\cdot)}^{*}:[t-h, t] \times \mathbf{C}^{n} \times[-h, 0] \rightarrow \mathbf{C}^{n}$, defined by

$$
\begin{aligned}
& x_{t}^{*}(\phi, \theta)=\left(T_{s}(t, 0) \phi\right)(\theta) \\
&:= T(t+\theta) \phi\left(0^{+}\right) \\
&+\int_{-h}^{0} T(t+\theta, \tau) \phi(\tau) U(\tau) d \tau, \quad \forall \theta \in[-h, 0], t \geq h, \\
&\left(T_{S}(0,0) \phi\right)(\theta)=x_{0}(\phi, \theta)=\phi(\theta), \quad \forall \theta \in[-h, 0],
\end{aligned}
$$

with $x(0)=\phi(0)$ is the unique solution of the limiting equation (2.1), and the kernel $K(t, \cdot)$ : $[0, t] \rightarrow \mathbf{C}^{n}$ of $T_{s}(t, 0), \forall t \in \mathbf{R}_{0+}$ is defined by

$$
K(t, s)(\theta)=\int_{0}^{s} X(t+\theta-\tau) d \tau, \quad \forall \theta \in[-h, 0], \forall t \in \mathbf{R}_{0+},
$$

where $X$ is the fundamental matrix of (2.1) with initial values $X_{0}(0)=I_{n}$ and $X_{0}(\theta)=0$, $\forall \theta \in[-h, 0]$. The following technical result holds. 
Lemma 3.1. The following relations hold:

$$
\begin{gathered}
x_{t}=x_{t}(\phi, \theta)=x_{t}^{P_{\Lambda}}+x_{t}^{Q_{\Lambda}}, \quad \mathbf{R}_{+} \ni t \geq h, \\
x_{t}^{P_{\Lambda}}=\left(T_{s}(t, 0) \phi^{P_{\Lambda}}\right)(\theta)+\int_{0}^{t}\left(T_{S}(t, \tau) f\left(\tau, x_{\tau}\right)^{P_{\Lambda}}\right)(\theta) d \tau \\
=\left(T_{S}(t, 0) \phi^{P_{\Lambda}}\right)(\theta)+\int_{0}^{t}\left(T_{S}(t, \tau) X_{0}^{P_{\Lambda}}\right)(\theta) f\left(\tau, x_{\tau}\right) d \tau \\
=T(t+\theta, 0) \phi^{P_{\Lambda}}\left(0^{+}\right)+\int_{-h}^{0} T(t+\theta, \tau) \phi^{P_{\Lambda}}(\tau) U(\tau) d \tau \\
+\int_{0}^{t} T(t+\theta, \tau) X_{0}^{P_{\Lambda}} f\left(\tau, x_{\tau}\right) d \tau, \\
x_{t}^{Q_{\Lambda}}=\left(T_{S}(t, 0) \phi^{Q_{\Lambda}}\right)(\theta)+\int_{0}^{t}\left(T_{S}(t, \tau) f\left(\tau, x_{\tau}\right)^{Q_{\Lambda}}\right)(\theta) d \tau, \quad \mathbf{R}_{+} \ni t \geq h \\
=\left(T_{S}(t, 0) \phi^{Q_{\Lambda e}}\right)(\theta)+\int_{0}^{t}\left(d[K(t, \tau)]^{Q_{\Lambda}}\right)(\theta) f\left(\tau, x_{\tau}\right), \quad \mathbf{R}_{+} \ni t \geq h, \\
x_{t}=\left(T_{S}(t, 0)\left(\phi^{P_{\Lambda}}+\phi^{Q_{\Lambda e}}\right)\right)(\theta)+\int_{0}^{t}\left(T_{S}(t, \tau) X_{0}^{P_{\Lambda}}+\left(d[K(t, \tau)]^{Q_{\Lambda}}\right)\right)(\theta) f\left(\tau, x_{\tau}\right) d \tau, \\
\quad \mathbf{R}_{+} \ni t \geq h,
\end{gathered}
$$

where

$$
X_{0}^{P_{\Lambda}}=\Phi_{\Lambda} \Psi_{\Lambda}(0), \quad K(t, \tau)^{Q_{\Lambda}}=K(t, \tau)-\Phi_{\Lambda}\left(\Psi_{\Lambda}, K(t, \tau)\right)
$$
small:

Also, the following relations hold for $\phi \in C_{e}(-h)$, for all $\varepsilon \in \mathbf{R}_{+}$, being sufficiently

$$
\begin{gathered}
\left|T(t, 0) \phi^{P_{\Lambda}}\right| \leq M_{1} e^{(\mu-\varepsilon) t}\left|\phi^{P_{\Lambda}}\right|, \quad\left|T(t, 0) X_{0}^{P_{\Lambda}}\right| \leq M_{1} e^{(\mu-\varepsilon) t} ; \quad \forall t \in \mathbf{R}_{0-}, \\
\left|T(t, 0) \phi^{Q_{\Lambda e}}\right| \leq M_{1} e^{(\mu-\varepsilon) t}\left|\phi^{Q_{\Lambda e}}\right|, \quad \forall t \in \mathbf{R}_{0+},
\end{gathered}
$$

for some $M_{1}=M_{1}(\varepsilon) \in \mathbf{R}_{+}$irrespective of the multiplicity of the eigenvalue of the limiting equation (2.1) whose real part is $\mu$. 
Proof. Equations (3.5)-(3.7) hold for any $\mathbf{R}_{0+} \ni t \geq h$ from Theorem 2.3, the definition of the set $\Lambda$ in Theorem 2.1, (2.20), Lemma 2.2, and (2.46)-(2.49). To obtain (3.5)-(3.7), the following identities are used:

$$
\begin{aligned}
\int_{0}^{t+\theta}\left(T_{S}(t, \tau) f\left(\tau, x_{\tau}\right)^{P_{\Lambda}}\right)(\theta) d \tau & =\int_{0}^{t}\left(T_{s}(t, \tau) X_{0}^{P_{\Lambda}}\right)(\theta) f\left(\tau, x_{\tau}\right) d \tau \\
& =\int_{0}^{t} T(t+\theta, \tau) X_{0}^{P_{\Lambda}} f\left(\tau, x_{\tau}\right) d \tau \\
\int_{0}^{t}\left(T_{S}(t, \tau) f\left(\tau, x_{\tau}\right)^{P_{\Lambda}}\right)(\theta) d \tau & =\int_{0}^{t+\theta}\left(T_{S}(t, \tau) f\left(\tau, x_{\tau}\right)^{P_{\Lambda}}\right)(\theta) d \tau \\
& =\int_{0}^{t}\left(T_{s}(t, \tau) X_{0}^{P_{\Lambda}}\right)(\theta) f\left(\tau, x_{\tau}\right) d \tau
\end{aligned}
$$

since $f\left(\tau, x_{\tau}\right)=0$ for $\tau<0$ and $T(t, \tau)=0$ for $\tau>t$ and $\theta \in[-h, 0]$. Equation (3.8) follows directly by substitution of (3.6), and (3.7) into (3.5). The norm relations (3.9) hold directly from (3.5)-(3.7) through (3.8).

It turns out that for any $\phi \in C_{e}(-h)$, the above relations hold also for any $t \in \mathbf{R}_{-h}$ by replacing $Q_{\Lambda} \rightarrow Q_{\Lambda e}$ in (3.5)-(3.7). Equation (3.9) also holds for $t \in \mathbf{R}_{-h}$ since $\phi \in C_{e}(-h)$ is bounded. The second relation in (3.5) may be rewritten as $\left|T(t, 0) \phi^{Q_{\Lambda}}\right| \leq M_{1} e^{(\mu-\varepsilon) t}$ for any $\mathbf{R}_{0+} \ni t \geq h$ and extended to any $t \in \mathbf{R}_{0+}$ if $\phi$ is continuous on its definition domain [- $\left.h, 0\right]$. A direct consequence of Lemma 3.1, (3.8) and Proposition 2.5 is that if $x_{t}^{P_{\Lambda}}=\Phi_{\Lambda} u(t)$, for all $t \in \mathbf{R}_{0+}$ then $u$ is a solution of the ordinary differential equation $\dot{u}(t)=B_{\Lambda} u(t)+\Psi_{\Lambda}(0) f\left(t, x_{t}\right)$, [28], which is given explicitly by:

$$
\begin{aligned}
u(t) & =e^{B_{\Lambda} t}\left(u(0)+\int_{0}^{t} e^{-B_{\Lambda} \tau} \Psi_{\Lambda}(0) f\left(\tau, x_{\tau}\right) d \tau\right) \\
& =e^{B_{\Lambda} t} u(0)+\int_{0}^{t} e^{B_{\Lambda}(t-\tau)} \Psi_{\Lambda}(0) f\left(\tau, x_{\tau}\right) d \tau \\
& =\Phi_{\Lambda}^{-1} T(t, 0) \Phi_{\Lambda} u(0)+\int_{0}^{t} \Phi_{\Lambda}^{-1} T(t, \tau) \Phi_{\Lambda} \Psi_{\Lambda}(0) f\left(\tau, x_{\tau}\right) d \tau \\
& =\Phi_{\Lambda}^{-1}\left(T(t, 0) \Phi_{\Lambda} u(0)+\int_{0}^{t} T(t, \tau) \Phi_{\Lambda} \Psi_{\Lambda}(0) f\left(\tau, x_{\tau}\right) d \tau\right)
\end{aligned}
$$

for $u(0)=\int_{-h}^{0} e^{B_{\Lambda}(t-\tau)} \phi(\tau) d \tau$ so that

$$
\begin{aligned}
x_{t}^{P_{\Lambda}} & =\Phi_{\Lambda} u(t) \\
& =T(t, 0) \Phi_{\Lambda} u(0)+\int_{0}^{t} T(t, \tau) \Phi_{\Lambda} \Psi_{\Lambda}(0) f\left(\tau, x_{\tau}\right) d \tau \\
\dot{u}(t) & =B_{\Lambda} \Phi_{\Lambda}^{-1} T(t, 0) \Phi_{\Lambda} u(0)+\int_{0}^{t} B_{\Lambda} \Phi_{\Lambda}^{-1} T(t, \tau) \Phi_{\Lambda} \Psi_{\Lambda}(0) f\left(\tau, x_{\tau}\right) d \tau+\Psi_{\Lambda}(0) f\left(t, x_{t}\right) \\
& =\Phi_{\Lambda}^{-1}\left(\dot{T}(t, 0) \Phi_{\Lambda} u(0)+\int_{0}^{t} \dot{T}(t, \tau) \Phi_{\Lambda} \Psi_{\Lambda}(0) f\left(\tau, x_{\tau}\right) d \tau\right)+\Psi_{\Lambda}(0) f\left(t, x_{t}\right) .
\end{aligned}
$$


It is now proved that the asymptotic difference function between some eigensolution of (2.1), that is, a finite sum of solutions of (2.1) corresponding to the set $\Lambda_{0}(\mu)$ of the form $p(t) e^{\lambda t}$, where $p$ is a $\mathbf{C}^{n}$-valued polynomial and $\lambda \in \Lambda_{0}(\mu)$, and some corresponding solution of (2.2)(2.3) grows non faster than linearly with the norm of the solution of the limiting equation (2.1), [32]. If $\gamma(t)$ converges to zero exponentially then the asymptotic difference function between both solutions has strict Lyapunov exponent smaller than that of the corresponding limiting eigensolution. As a result, a solution of (2.2)-(2.3) is of the same exponential order as that of its limiting equation for sufficiently large time. Define the set of distinct eigenvalues of the limiting equation (2.1) as

$$
\mathrm{CE}:=\left\{\lambda_{j \mu_{i}} \in \mathbf{C}: \operatorname{Re} \lambda_{j \mu_{i}}=\mu_{i}, \operatorname{det}\left(\widehat{T}^{-1}\left(\lambda_{\mu_{i}}\right)\right)=0 ; \forall j \in \operatorname{In}_{\mathrm{CEi}} \subset \mathbf{Z}_{+}, \forall i \in \operatorname{In}_{\mathrm{CER}} \subset \mathbf{Z}_{+}\right\}
$$

It is obvious that the cardinal of CE may be infinity (since (2.1) is a functional differential equation), but always numerable. The also denumerable subsets $\operatorname{In}_{\mathrm{CEi}}$ and $\operatorname{In}_{\mathrm{CER}}$ of the set of positive integers are indicator sets for all distinct members of $\mathrm{CE}$ with real part $\mu_{i}$ and for all the distinct real parts of the members of CE, respectively. It follows that $\operatorname{Card}(\mathrm{CE})=\sum_{i \in \operatorname{In}_{\mathrm{CER}}}$ Card $\operatorname{In}_{\mathrm{CEi}} \leq \chi_{0}$. The total number of eigenvalues taking account for their multiplicities $v_{i j} ; \forall j \in \operatorname{In}_{\mathrm{CEi}}, \forall i \in \operatorname{In}_{\mathrm{CER}}$, is

$$
\vartheta=\sum_{i \in \operatorname{In}_{\mathrm{CER}}} \sum_{j \in \operatorname{In}_{\mathrm{CEi}}} v_{i j} \text { Card } \operatorname{In}_{\mathrm{CEi}} \geq \operatorname{Card}(\mathrm{CE})=\sum_{i \in \operatorname{In}_{\mathrm{CER}}} \operatorname{Card} \operatorname{In}_{\mathrm{CEi}} .
$$

In particular, $\operatorname{Card}(\mathrm{CE})=\chi_{0}$ ( $\chi_{0}$ standing for infinity denumerable cardinal as opposite to a non-numerable infinity cardinal typically denoted by $\infty$ ) or Card(CE) is finite. The above definition relies on the fact distinct (non real) eigenvalues $\lambda_{j \mu_{i}}$ of (2.1), $\forall j \in \operatorname{In}_{\mathrm{CEi}}$ can have identical real part. Similar sets of eigenvalues of (2.1) as those in (2.18)-(2.20) may be defined being associated to each member of $\mathrm{CE}$ as follows:

$$
\begin{aligned}
\Lambda_{0}\left(\mu_{i}\right) & :=\left\{\lambda \in \mathrm{CE}: \operatorname{Re} \lambda=\mu_{i}\right\} ; \\
\Lambda_{1}\left(\mu_{i}\right) & :=\left\{\lambda \in \mathrm{CE}: \operatorname{Re} \lambda>\mu_{i}\right\} ; \\
\Lambda\left(\mu_{i}\right) & =\Lambda_{0}\left(\mu_{i}\right) \cup \Lambda_{1}\left(\mu_{i}\right) \\
& :=\left\{\lambda \in \mathrm{CE}: \operatorname{Re} \lambda \geq \mu_{i}\right\} .
\end{aligned}
$$

The following result holds concerning an asymptotic comparison of eigensolutions of (2.1) with the corresponding associated solution of (2.2) and (2.3) under a special form of the perturbation function.

Theorem 3.2. Suppose that at least one $\lambda_{\mu} \in C E$ has real part $\mu$ being identical strict Lyapunov exponent of some solution of (2.2) under (2.3). Suppose also that $f\left(t, x_{t}\right)$ satisfies the hypotheses of Theorem 2.1. Then, a solution of (2.2)-(2.3) satisfies $x(t)=y(t)+O\left(e^{c t}\right), \forall t \in \mathbf{R}_{0+}$ and any $\mathbf{R} \ni c>\mu$. Also, $x(t)=y(t)+O\left(t^{\nu-1} e^{\mu t}\right)$ for any $f\left(t, x_{t}\right)$ satisfying the hypotheses of Theorem 2.1 with $v$ being the largest multiplicity among those of all distinct $\lambda_{\mu} \in C E$.

Assume, in addition, that $K_{0 \alpha}=0$ for any norm $\alpha$ and $\gamma: \mathbf{R}_{0+} \rightarrow \mathbf{R}_{0+}$ satisfies $\gamma(t)=$ $o\left(e^{-a t}\right)$ as $t \rightarrow \infty$ for some $a \in \mathbf{R}_{+}$(i.e., $\gamma(t) \rightarrow 0$ as $t \rightarrow \infty$ exponentially). Then, $\exists \varepsilon \in \mathbf{R}_{+}$ 
Abstract and Applied Analysis

and a nontrivial eigensolution of (2.1) corresponding to the set $\Lambda_{0}(\mu)$ such that $x(t)=y(t)+$ $o\left(e^{(\mu-\varepsilon) t}\right)$ as $t \rightarrow \infty$.

Proof. Note that for any norm $\alpha, x_{t}=O\left(\left|x_{t}^{P_{0}\left(\mathbf{R}_{-h}\right)}\right|_{\alpha}\right) ; \forall t \in \mathbf{R}_{0+}$ [Theorem 2.3(ii)] with $C_{e}\left(\mathbf{R}_{-h}\right)=P_{\Lambda}\left(\mathbf{R}_{-h}\right) \oplus Q_{\Lambda e}\left(\mathbf{R}_{-h}\right), P_{\Lambda}\left(\mathbf{R}_{-h}\right)=P_{\Lambda_{0}}\left(\mathbf{R}_{-h}\right) \oplus P_{\Lambda_{1}}\left(\mathbf{R}_{-h}\right)$ with $P_{\Lambda_{0}}\left(\mathbf{R}_{-h}\right)$ being the eigenspace associated with $\Lambda_{0}(\mu)$ for $t \in \mathbf{R}_{-h}$. For $t \geq h$, the related direct sum decomposition $C\left(\mathbf{R}_{0+}\right)=P_{\Lambda}\left(\mathbf{R}_{0+}\right) \oplus Q_{\Lambda}\left(\mathbf{R}_{0+}\right), P_{\Lambda}\left(\mathbf{R}_{0+}\right)=P_{\Lambda_{0}}\left(\mathbf{R}_{0_{+}}\right) \oplus P_{\Lambda_{1}}\left(\mathbf{R}_{0+}\right)$ since may be used for the eigensolutions of (2.1) since the solution is time-differentiable for $t \in \mathbf{R}_{0+}$. Thus, $x(t)=O\left(e^{\mathrm{ct}}\right)$ and $y(t)=O\left(e^{\mathrm{ct}}\right)$ so that $x(t)-y(t)=O\left(e^{\mathrm{ct}}\right)$ for any given perturbation function $f\left(t, x_{t}\right)$ satisfying the constraints of Theorem 2.1 and all $\mathbf{R} \ni c>\mu=\operatorname{Re} \lambda_{\mu}, \lambda_{\mu} \in \mathrm{CE}$.

If all $\lambda_{\mu} \in \mathrm{CE}$ are simple then $x(t)-y(t)=O\left(e^{\mathrm{ct}}\right), \mathbf{R} \ni c \geq \mu=\operatorname{Re} \lambda_{\mu}$ for any given perturbation function $f\left(t, x_{t}\right)$ satisfying the constraints of Theorem 2.1.

Otherwise (i.e., at least one $\lambda_{\mu} \in \mathrm{CE}$ is not simple), $x(t)-y(t)=O\left(p_{\lambda_{u}}(t) e^{\mu t}\right)=O\left(e^{\mathrm{ct}}\right)$

for some $\mathbf{R}_{-} \ni c>\mu$ with $p_{\lambda_{u}}:[0, t] \rightarrow \mathbf{C}^{n}$ being a polynomial of degree $v$ equating the largest multiplicity among those of all distinct $\lambda_{\mu} \in \mathrm{CE}$.

The first part of the result has been fully proved. Now, note that (3.8) in Lemma 3.1 can be equivalently rewritten as

$$
x_{t}-y_{t}=\int_{0}^{t}\left(T_{s}(t, \tau) X_{0}^{P_{\Lambda}}+\left(d[K(t, \tau)]^{Q_{\Lambda}}\right)\right)(\theta) f\left(\tau, x_{\tau}\right) d \tau, \quad \mathbf{R}_{+} \ni t \geq h .
$$

Also, if $\gamma(t)=o\left(e^{-a t}\right)$ as $t \rightarrow \infty$ and since $\left|x_{t}\right|=o\left(e^{(\mu+\varepsilon) t}\right)$ as $t \rightarrow \infty$, irrespective of the multiplicity of $\lambda_{\mu}$, from the definition of the strict Lyapunov exponent, one gets by using $\gamma(\tau)\left|x_{\tau}\right| \leq M_{\gamma} M_{x} e^{(\mu+\varepsilon-\alpha) \tau}$ from (2.8) with $K_{0 \alpha}=0\left(\right.$ since $\gamma(t)=o\left(e^{- \text {at }}\right) \rightarrow 0$ as $\left.t \rightarrow \infty\right)$ and (3.10b):

$$
\begin{aligned}
\left|\int_{0}^{t}\left(d[K(t, \tau)]^{Q_{\Lambda}}\right)(\theta) f\left(\tau, x_{\tau}\right) d \tau\right|_{\alpha} & \leq M_{2}(\alpha) e^{(\mu-\varepsilon) t} \int_{0}^{\infty} e^{(2 \varepsilon-\alpha) \tau} d \tau \\
\leq & \frac{M_{2}(\alpha)}{\alpha-2 \varepsilon} e^{(\mu-\varepsilon) t}=o\left(e^{(\mu-\varepsilon) t}\right), \quad \forall t \in \mathbf{R}_{0+} \\
\left|\int_{0}^{t} T_{S}(t, \tau) X_{0}^{P_{\Lambda}}(\theta) f\left(\tau, x_{\tau}\right) d \tau\right|_{\alpha} & \leq M_{3}(\alpha) e^{(\mu-\varepsilon) t} \int_{0}^{\infty} e^{(2 \varepsilon-\alpha) \tau} d \tau \\
& \leq \frac{M_{2}(\alpha)}{\alpha-2 \varepsilon} e^{(\mu-\varepsilon) t}=o\left(e^{(\mu-\varepsilon) t}\right), \quad \forall t \in \mathbf{R}_{0+}
\end{aligned}
$$

since $\left\|X_{0}^{P_{\Lambda}}(\theta)\right\|_{\alpha}=\left\|\Phi_{\Lambda} \Psi_{\Lambda}(\theta)\right\|_{\alpha}=\left\|\Phi_{\Lambda}(0) \Psi_{\Lambda}(0) e^{2 B_{\Lambda} \theta}\right\|_{\alpha}<\infty, \forall \theta \in[-h, 0]$ provided that $0<$ $\varepsilon<\alpha / 2$. Then, $x_{t}-y_{t}=o\left(e^{(\mu-\varepsilon) t}\right)$ as $t \rightarrow \infty, \forall \varepsilon \in \mathbf{R}_{+}$satisfying $0<\varepsilon<\alpha / 2$.

Remark 3.3. An important observation follows. Note that the error between any eigensolutions of the limiting equation (2.1) and the associated solution of (2.2)-(2.3) satisfying $x_{t}-y_{t}=o\left(e^{(\mu-\varepsilon) t}\right)$ as $t \rightarrow \infty$ if $\gamma(t)=o\left(e^{-a t}\right)$ as $t \rightarrow \infty$ and $K_{0 \alpha}=0$ for sufficiently small $\varepsilon \in \mathbf{R}_{+}$(proven in Theorem 3.2) does not imply that $x_{t}=o\left(e^{(\mu-\varepsilon) t}\right), y_{t}=o\left(e^{(\mu-\varepsilon) t}\right)$ as $t \rightarrow \infty$ if $\gamma(t)=o\left(e^{- \text {at }}\right)$ as $t \rightarrow \infty$ for sufficiently small $\varepsilon \in \mathbf{R}_{+}$.

The asymptotic behaviors if $\gamma(t)=o\left(e^{- \text {at }}\right)$ and $K_{0 \alpha}=0$ as $t \rightarrow \infty$ are as follows:

$$
y_{t}=o\left(e^{\mu t}\right), \quad x_{t}=y_{t}+o\left(e^{(\mu-\varepsilon) t}\right)=o\left(e^{\mu t}\right)+o\left(e^{(\mu-\varepsilon) t}\right)=o\left(e^{\mu t}\right), \quad \text { as } t \rightarrow \infty,
$$


for sufficiently small $\varepsilon \in \mathbf{R}_{+}$if $\lambda_{\mu} \in \mathrm{CE}$ is real simple or there are two $\lambda_{\mu_{1,2}} \in \mathrm{CE}$ simple complex conjugate ones. In the above, equations, $y_{t}=o\left(e^{\mu t}\right), x_{t}=o\left(e^{\mu t}\right)$ hold even if $K_{0 \alpha} \neq 0$ provided that $\lambda_{\mu}$ fulfils some of the above constraints. Furthermore,

$$
y_{t}=o\left(e^{\mathrm{ct}}\right), \quad x_{t}=y_{t}+o\left(e^{(\mu-\varepsilon) t}\right)=o\left(e^{\mathrm{ct}}\right)+o\left(e^{(\mu-\varepsilon) t}\right)=o\left(e^{\mathrm{ct}}\right), \quad \text { as } t \rightarrow \infty,
$$

for sufficiently small $\varepsilon \in \mathbf{R}_{+}$and all real $c>\mu=\operatorname{Re} \lambda_{\mu}$ if $\lambda_{\mu} \in C E$ is multiple (i.e., of multiplicity greater then unity). Also, $x_{t}-y_{t}=O\left(e^{c t}\right) \Rightarrow x_{t}=O\left(e^{c t}\right) \wedge y_{t}=O\left(e^{c t}\right), t \in \mathbf{R}_{0+}$, $c>\mu=\operatorname{Re} \lambda_{\mu}$ irrespective of the multiplicity of $\lambda_{\mu}$ for any generic perturbation function $f\left(t, x_{t}\right)$ satisfying the hypothesis of Theorem 2.1. However, the above implication is true for any arbitrary such a function and $c=\mu$, only if $\lambda_{\mu}$ is an eigenvalue of multiplicity unity of the limiting equation (2.1).

Remark 3.3.

Theorem 3.2 leads directly to the subsequent stability result by taking also into account

Corollary 3.4. Suppose that there exists a (nonnecessarily unique) $\lambda_{\mu} \in \mathrm{CE}$ with $\mu<0$ being the strict Lyapunov exponent of some solution of (2.2) under (2.3) and that $\Lambda_{0}(\mu)=\Lambda(\mu)$; that is, $\Lambda_{1}(\mu)=\varnothing$. Suppose also that $f\left(t, x_{t}\right)$ satisfies the hypotheses of Theorem 2.1 and, furthermore, $K_{0 \alpha}=$ 0 (for any $\alpha$-norm) and $\gamma(t)=o\left(e^{-a t}\right)$ as $t \rightarrow \infty$ for some $a \in \mathbf{R}_{+}$. Then, $\exists \varepsilon \in \mathbf{R}_{+}$such that any solutions of (2.1) and (2.2)-(2.3) satisfy:

$x(t)=y(t)+o\left(e^{-(|\mu|+\varepsilon) t}\right) \longrightarrow y(t) \longrightarrow o\left(t^{\nu-1} e^{-|\mu| t}\right) \longrightarrow o\left(e^{-|c| t}\right) \longrightarrow 0 \quad$ exponentially as $t \longrightarrow \infty$,

for some $\mathbf{R}_{-} \ni c \geq \mu$ with $v$ being equal to the largest multiplicity of among those of all distinct $\lambda_{\mu} \in \mathrm{CE}$. The above relations hold with $c=\mu$ if and only if all the eigenvalues of (2.1) satisfying the given assumptions are simple. As a result, both functional equations (2.2)-(2.3) and its limiting one are globally asymptotically Lyapunov stable with exponential stability.

Proof. Theorem 3.2 applies for $c>\mu$ with $\left|\lambda_{\mu}\right|$ being the spectral radius and $\mu<0$ the spectral (or stability) abscissa, that is, there is no member of CE with real part to the right of $\mu<$ 0 since $\Lambda_{1}(\mu)=\varnothing$. If all such $\lambda_{\mu} \in \mathrm{CE}$ are simple then the result applies also for $c \geq \mu$ (see Remark 3.3). Define the bounded real nonnegative function $V:\left[t_{0}, \infty\right) \times \mathbf{C}^{n} \rightarrow \mathbf{R}_{0+}$ as $V(t, x(t)):=\|x(t)\|_{2}^{2}$ for any finite $t_{0} \in \mathbf{R}_{0+}$. Note that $V(t, 0)=0$ and $V(t, x(t))$ are strictly monotonically increasing with $\|x(t)\|$. It follows from (2.2)-(2.3) that $\exists \dot{V} \in P C^{(1)}\left(\left[t_{0}, \infty\right) \times\right.$ $\left.\mathbf{C}^{n}, \mathbf{R}\right)$, point-wise defined by $\dot{V}(t, x(t)):=2 \dot{x}^{*}(t) x(t)$, and

$$
\begin{aligned}
0 \leq V(t, x(t)) & =V\left(t_{0}, x\left(t_{0}\right)\right)+\int_{t 0}^{t} \dot{V}(\tau, x(\tau)) d \tau \\
& \leq K e^{-2|c|\left(t-t_{0}\right)} \longrightarrow 0 \text { exponentially as } t \longrightarrow \infty
\end{aligned}
$$

Then, $\exists \lim _{t \rightarrow \infty} \int_{t 0}^{t} \dot{V}(\tau, x(\tau)) d \tau=2 \lim _{t \rightarrow \infty} \int_{t 0}^{t} \dot{x}^{*}(\tau) x(\tau) d \tau=-V\left(t_{0}, x\left(t_{0}\right)\right) \leq 0$ which is finite since $t_{0}$ is finite and the solution to (2.2)-(2.3) is everywhere continuous on its definition domain. By assuming $\neg \exists t_{0} \in \mathbf{R}_{0+}$ such that $\dot{V}(t, x(t)) \leq 0, t \in\left[t_{0}, \infty\right)$ then $\neg \exists \lim _{t \rightarrow \infty} \int_{t_{0}}^{t} \dot{V}$ 
$(\tau, x(\tau)) d \tau=-V\left(t_{0}, x\left(t_{0}\right)\right) \leq 0$ which is a contradiction to the already proven existence of such a limit. As a result, $\exists t_{0}$ (being sufficiently large but finite) $\in \mathbf{R}_{0+}$ such that $\dot{V}(t, x(t)) \leq 0$, $\forall t \in\left[t_{0}, \infty\right)$ and $\dot{V}(t, x(t))<0$ on some (non-necessarily connected) subinterval of infinite measure of $\left[t_{0}, \infty\right)$ with $\dot{V}(t, x(t)) \rightarrow 0$ as $t \rightarrow \infty$ then necessarily there is a connected terminal subinterval $\left[t_{0}^{\prime}, \infty\right) \subset\left[t_{0}, \infty\right)$ of infinite measure where $\dot{V}(t, x(t))<0$. Thus, $V(t, x(t))$ is nonnegative and converges exponentially to zero with nonpositive time-derivative which also converges to zero within some interval of infinite measure. As a result, $V(t, x(t))$ is a Lyapunov function with negative time-derivative within a connected real interval of infinite measure which has zero limit.

Note that the limiting differential functional equation is, furthermore, globally uniformly asymptotically Lyapunov stable under the asymptotic stability conditions of Corollary 3.4. Note that $K_{0 \alpha}>0$ (for any $\alpha$-norm) in (2.3) implies that $K_{0 \alpha \prime}>0$ for any other norm $\alpha^{\prime}$ and conversely. Also, $K_{0 \alpha}=0 \Leftrightarrow K_{0 \alpha \prime}=0$. The above result is concerned with global asymptotic stability with exponential decay rate. Global uniform Lyapunov stability (i.e. boundedness of solutions with a common upper-bound for all time for any bounded function of initial conditions) holds under weaker conditions; that is, $K_{0 \alpha}>0$ and $\mu=0$ if $\lambda_{\mu} \in \mathrm{CE}$ associated with the strict Lyapunov exponent have unity multiplicities or if they have any multiplicities but $K_{0 \alpha}=0$. The precise related stability result follows which proofs follows directly from Corollary 3.4 and Theorem 3.2.

Corollary 3.5. Suppose that there exists at least one $\lambda_{\mu} \in \mathrm{CE}$ with $\mu \leq 0$ being the strict Lyapunov exponent of some solution of (2.2) under (2.3) and that $\Lambda_{0}(\mu)=\Lambda(\mu)$; that is, $\Lambda_{1}(\mu)=\varnothing$. Suppose also that $f\left(t, x_{t}\right)$ satisfies the hypotheses of Theorem 2.1 with $K_{0 \alpha} \geq 0$ (for any $\alpha$-norm) and $\gamma(t)=$ $o\left(e^{-a t}\right)$ as $t \rightarrow \infty$ for some $a \in \mathbf{R}_{+}$. Then, $\exists \varepsilon \in \mathbf{R}_{+}$such that any solutions of (2.1) and (2.2)-(2.3) satisfy

$$
x(t)=y(t)+O\left(e^{-|\mu| t}\right) \longrightarrow y(t) \longrightarrow O\left(e^{-|\mu| t}\right), \quad \forall t \in \mathbf{R}_{0+} .
$$

As a result, the limiting equation (2.1) as well as (2.2)-(2.3) are both globally uniformly Lyapunov stable if all $\lambda_{\mu} \in \mathrm{CE}$ have any multiplicities and $\mu<0$ or if they have all unity multiplicities and $\mu=0$. Equations (2.1) and (2.2)-(2.3) are both globally Lyapunov asymptotically stable with exponential decay rate if $\mu<0$ and $K_{0 \alpha}=0$, satisfying:

$$
\begin{array}{r}
x(t)=y(t)+o\left(e^{-|\mu| t}\right) \longrightarrow y(t) \longrightarrow o\left(p_{\lambda_{u}}(t) e^{-|\mu| t}\right) \longrightarrow o\left(e^{-|c| t}\right) \longrightarrow 0 \\
\text { exponentially as } t \longrightarrow \infty,
\end{array}
$$

for some $\mathbf{R}_{-} \ni c \geq \mu$ with $p_{\lambda_{u}}:[0, t] \rightarrow \mathbf{C}^{n}$ being a polynomial of degree equal to the largest multiplicity of all such distinct $\lambda_{\mu} \in \mathrm{CE}$. The above relations hold with $c=\mu$ if and only if all the eigenvalues of (2.1) satisfying the given assumptions are simple, [32].

A parallel result to Theorem 3.2 obtained under weaker conditions on the perturbation function (2.3) follows.

Theorem 3.6. Suppose that at least one $\lambda_{\mu} \in \mathrm{CE}$ has real part $\mu$ being identical strict Lyapunov exponent of some solution of (2.2) under (2.3). Suppose also that $f\left(t, x_{t}\right)$ satisfies the hypotheses of Theorem 2.1. Suppose, in addition, that $K_{0 \alpha}=0$ for any norm $\alpha$, and $\gamma: \mathbf{R}_{0+} \rightarrow \mathbf{R}_{0+}$ satisfies either 
$\int_{0}^{\infty} t^{v-1} \gamma(t) d t<\infty$ or $\gamma(t) \leq o\left(t^{1-v}\right)$ as $t \rightarrow \infty$, with the inequality being strict if $v=1$, where $v$ is the largest multiplicity among those of all distinct $\lambda_{\mu} \in \mathrm{CE}$. Then, $\exists \varepsilon \in \mathbf{R}_{+}$and a nontrivial eigensolution of (2.1) corresponding to the set $\Lambda_{0}(\mu)$ such that $x(t)=y(t)+o\left(e^{\mu t}\right)$ as $t \rightarrow \infty$.

Furthermore, $x(t)=o\left(t^{\nu-1} e^{\mu t}\right)$ and $y(t)=o\left(t^{\nu-1} e^{\mu t}\right)$.

Proof. One gets for sufficiently small $\varepsilon \in \mathbf{R}_{+}$from $\int_{0}^{t} t^{\nu-1} \gamma(t) d t<\infty$ and Theorem 3.2, (3.18)(3.19) and $K_{0 \alpha}=0$ :

$$
\begin{aligned}
\left|\int_{0}^{t}\left(d[K(t, \tau)]^{Q_{\Lambda}}\right)(\theta) f\left(\tau, x_{\tau}\right) d \tau\right|_{\alpha} & \leq M_{4}(\alpha) e^{\mu t} \int_{0} \infty \frac{e^{-\mu \tau} e^{(\varepsilon-\mu) \tau} e^{(\varepsilon+\mu) \tau}}{\tau^{(\nu-1) / 2}}\left(\tau^{(\nu-1) / 2} \gamma(\tau)\right) d \tau \\
& \leq M_{4}(\alpha) e^{\mu t}\left[\int_{0}^{\infty}\left(\frac{e^{2(2 \varepsilon-\mu) \tau}}{\tau^{\nu-1}}\right) d \tau\right]^{1 / 2}\left[\int_{0}^{\infty} \tau^{\nu-1} \gamma^{2}(\tau) d \tau\right]^{1 / 2} \\
& \leq M_{5}(\alpha) e^{\mu t}\left[\int_{0}^{\infty}\left(\frac{e^{2(2 \varepsilon-\mu) \tau}}{\tau^{\nu-1}}\right) d \tau\right]^{1 / 2}, \quad \forall t \in \mathbf{R}_{0+},
\end{aligned}
$$

where $M_{5}(\alpha) \geq M_{4}(\alpha) \operatorname{Sup}_{0 \leq t<\infty}(\gamma(t))$ which is a finite real constant since $\gamma(t)$ is continuous on $\mathbf{R}_{0+}$ and has zero limit as $t \rightarrow \infty$ so that it is uniformly bounded. Now, if $\mu \in \mathbf{R}_{+}$for $\mathbf{R}_{+} \ni \varepsilon \in(0, \mu / 2), \mathbf{R}_{-} \ni d>b:=(2 \varepsilon-\mu) v \in \mathbf{R}_{-}$and $\mathbf{R}_{0+} \ni t \geq t_{0}:=e^{(d-b) /(v-1)}$ :

$$
\begin{gathered}
\int_{t_{0}}^{\infty}\left(\frac{e^{2(2 \varepsilon-\mu) \tau}}{\tau^{\nu-1}}\right) d \tau \leq \int_{t_{0}}^{\infty} e^{-|d| \tau} d \tau \leq \frac{1}{|d|}=(\mu-2 \varepsilon) \mathcal{v}<\infty, \\
\int_{0}^{t_{0}}\left(\frac{e^{2(2 \varepsilon-\mu) \tau}}{\tau^{\nu-1}}\right) d \tau \leq M_{6}<\infty
\end{gathered}
$$

since $t_{0}$ is finite. Now from (3.27) into (3.26):

$$
\left|\int_{0}^{t}\left(d[K(t, \tau)]^{Q_{\Lambda}}\right)(\theta) f\left(\tau, x_{\tau}\right) d \tau\right|_{\alpha} \leq M_{5}(\alpha)\left(\sqrt{M_{6}}+\sqrt{(\mu-2 \varepsilon) v}\right) e^{\mu t}=o\left(e^{\mu t}\right) \text { as } t \longrightarrow \infty
$$

if $\mu \in \mathbf{R}_{+}$. If $\mu \in \mathbf{R}_{0-}$ then (3.10a) and (3.10b) of Lemma 3.1 may be replaced for $\mu \in \mathbf{R}_{0-}$ by taking $\varepsilon=0$ with

$$
\begin{gathered}
\left|T(t, 0) \phi^{P_{\Lambda}}\right| \leq M_{1} t^{\nu-1} e^{-|\mu| t}\left|\phi^{P_{\Lambda}}\right|, \quad\left|T(t, 0) X_{0}^{P_{\Lambda}}\right| \leq M_{1} t^{\nu-1} e^{-|\mu| t} ; \quad \forall t \in \mathbf{R}_{0-}, \\
\left|T(t, 0) \phi^{Q_{\Lambda e}}\right| \leq M_{1} t^{\nu-1} e^{-|\mu| t}\left|\phi^{Q_{\Lambda e}}\right| ; \quad \forall t \in \mathbf{R}_{0+} .
\end{gathered}
$$


By using (3.30), (3.26) may be replaced $\mu \in \mathbf{R}_{0-}$ with

$$
\begin{aligned}
\left|\int_{0}^{t}\left(d[K(t, \tau)]^{Q_{\Lambda}}\right)(\theta) f\left(\tau, x_{\tau}\right) d \tau\right|_{\alpha} & \leq M_{4}(\alpha) \int_{0}^{t} e^{-|\mu|(t-\tau)}\left(\tau^{\nu-1} \gamma(\tau)\right) d \tau \\
& \leq M_{4}(\alpha) \int_{0}^{\infty} \tau^{\nu-1} \gamma(\tau) d \tau \leq M_{7}(\alpha)<\infty, \quad \forall t \in \mathbf{R}_{0+} .
\end{aligned}
$$

On the other hand, $\int_{0}^{\infty} t^{\nu-1} \gamma(\tau) d \tau<\infty \Rightarrow \gamma(t)=o\left(1 / t^{\nu-1}\right)$ as $t \rightarrow \infty$ so that one gets for $\mu \in \mathbf{R}_{0 \text { - }}$ from Theorem 3.2, (3.18), since the integrals in (3.27) are bounded:

$$
\begin{aligned}
& \left|\int_{0}^{t}\left(d[K(t, \tau)]^{Q_{\Lambda}}\right)(\theta) f\left(\tau, x_{\tau}\right) d \tau\right|_{\alpha} \\
& \quad \leq M_{4}(\alpha) e^{\mu t} \int_{0}^{t} \tau^{1-v} e^{\varepsilon(2 \tau-t)} d \tau \\
& \quad \leq 2 M_{4}(\alpha) e^{\mu t} \int_{0}^{2 t}\left(\frac{\tau}{2}\right)^{1-v} e^{\varepsilon(\tau-t)} d \tau \\
& \quad \leq 2 M_{4}(\alpha) e^{\mu t} \int_{0}^{\infty}\left(\frac{\tau}{2}\right)^{1-v} e^{\varepsilon(\tau-t)} d \tau \\
& \quad \leq M_{8}(\alpha) e^{\mu t}=o\left(e^{-|\mu| t}\right) \quad \text { as } t \rightarrow \infty, \text { irrespective of the norm } \alpha .
\end{aligned}
$$

As a final result, $\left|\int_{0}^{t}\left(d[K(t, \tau)]^{Q_{\Lambda}}\right)(\theta) f\left(\tau, x_{\tau}\right) d \tau\right|_{\alpha}=o\left(e^{\mu t}\right)$ as $t \rightarrow \infty, \forall \mu \in \mathbf{R}$, and, furthermore, $\left|\int_{0}^{t}\left(d[K(t, \tau)]^{Q_{\Lambda}}\right)(\theta) f\left(\tau, x_{\tau}\right) d \tau\right|_{\alpha}=O\left(e^{\mu t}\right), \forall \mu \in \mathbf{R}_{0-}, \forall t \in \mathbf{R}_{0+}$, irrespective of the norm $\alpha$. Similar properties follow under close proofs for $\left|\int_{0}^{t} T_{S}(t, \tau) X_{0}^{P_{\Lambda}}(\theta) f\left(\tau, x_{\tau}\right) d \tau\right|_{\alpha}$. Thus, $x_{t}-y_{t}=o\left(e^{\mu t}\right)$ as $t \rightarrow \infty$ by using the above results in (3.26) and $x_{t}-y_{t}=O\left(e^{\mu t}\right), \forall t \in \mathbf{R}_{0+}$. The properties $x(t)=o\left(t^{\nu-1} e^{\mu t}\right)$ and $y(t)=o\left(t^{\nu-1} e^{\mu t}\right)$ of the solutions of (2.1) and (2.2)-(2.3) under the given hypotheses follow directly by using the appropriate reasoning quoted from Remark 3.3.

Lyapunov stability properties in terms of boundedness of the solutions and their asymptotic or exponential convergence to the equilibrium obtained from Theorem 3.6 are immediate as given in the following direct result.

Corollary 3.7. Corollaries 3.4-3.5 also apply "mutatis-mutandis" under the assumptions of Theorem 3.6.

\section{Some Direct Consequences and Applications}

Some particular cases of the functional differential equations (2.1) and (2.2)-(2.3) are of interest concerning stability issues as follows. 


\subsection{Functional Differential Equations with Point Delays}

The functional differential equation (2.2) can be equivalently described in the absence of finite distributed delays and Volterra-type dynamics by the $\mathrm{n}$-the order system of $\mathrm{n}$ functional firstorder differential equations:

$$
\dot{x}(t)=\sum_{i=0}^{m} A_{i} x\left(t-h_{i}\right)+f\left(t, x_{t}\right),
$$

where $h_{0}=0$. Its limiting differential system is defined in the same way from (4.1) for a perturbation function $f\left(t, x_{t}\right) \equiv 0$. The generic form of such a function is given in (2.2) and can include nonzero dynamics of finite-distributed delays and Volterra-type dynamics. Thus, $\widehat{T}(s)=\left(s I_{n}-\sum_{i=0}^{m} A_{i} e^{-h_{i} s}\right)^{-1}$ everywhere the inverse exists. Remember for later use that a matrix-valued function $G: \mathbf{C}_{0+} \rightarrow \mathbf{C}^{r \times m}$ is in the Hardy space $H_{\infty}$ if it is analytic in $\mathbf{C}_{+}, \lim _{\sigma \rightarrow 0^{+}} G(\sigma+\mathbf{i} \omega)=G(\mathbf{i} \omega)$ for almost all $\omega \in \mathbf{R}_{0+}$ and $\|G\|_{\infty}:=\sup _{s \in \mathbf{C}_{0+}} \bar{\sigma}(G(s))=$ $\sup _{\omega \in \mathbf{R}} \bar{\sigma}(G(\mathbf{i} \omega))<\infty$ with $\bar{\sigma}(G)$ denoting the largest singular value of $G$. Also, $R H_{\infty}$ is a the subset of $H_{\infty}$ of real-rational matrix valued functions then being proper and stable (i.e., they have no more zeros than poles and all the poles are in $\mathbf{C}_{-}$), [33]. Note that the used norm notation $\|G\|_{\infty}$ for $G \in H_{\infty}$ is similar to the notation for $\ell_{\infty}$-matrix/vector norms but no confusion is expected from the different context of use. If $G(s)=G^{*}\left(s^{*}\right)$ then $\|G\|_{\infty}:=\sup _{\omega \in \mathbf{R}_{0+}} \bar{\sigma}(G(\mathbf{i} \omega))$.

The following result holds.

Theorem 4.1. The following properties hold.

(i) The limiting differential system associated with (4.1) is globally asymptotically stable independent of the delays (i.e., $h_{i} \in \mathbf{R}_{0+}(\forall i \in \bar{m})$ if $A_{0}$ is a stability matrix and there exists $\beta_{i} \in \mathbf{R}_{+}(\forall i \in \bar{m})$ such that $\sum_{i=1}^{m} \beta_{i}^{2}=1$, and

$$
\left\|\left(\mathbf{i} \omega I_{n}-\sum_{i=0}^{m} A_{i}\right)^{-1}\left[\beta_{1}^{-1} A_{1}, \ldots, \beta_{r}^{-1} A_{m}\right]\right\|_{2}<1, \quad \omega \in \mathbf{R}_{0+},
$$

or

$$
\left\|\left[\beta_{1}^{-1} A_{1}, \ldots, \beta_{r}^{-1} A_{m}\right]\right\|_{2}<-\kappa_{2}\left(A_{0}\right)
$$

This implies as a result that $\kappa_{2}\left(A_{0}\right)<0$ and that any matrix $\left(A_{0}+\sum_{i \in J_{k}} A_{i}\right)$ is a stability matrix, $\forall J_{k} \subseteq \bar{m}$, where $J_{\bar{m}}:=\bigcup_{k \in s_{\bar{m}}} J_{k}$ is the union of all the $s_{\bar{m}}$ denumerable sets obtained by combining members of $\bar{m}$ (i.e., $J_{\bar{m}}$ is the set of parts of $\bar{m}$ ).

(ii) If property (i) holds, then $\overline{A_{0}}=A_{0}+\rho I_{n}$ is a stability matrix for all $\mathbf{R}_{0+} \ni \rho \in\left[0, \rho_{0}\right)$ with $\rho_{0}:=1 /\left\|A_{0}^{-1}\right\|_{2}$. The differential system (4.1) is globally asymptotically stable independent of the size 
of the delays with stability (or spectral) abscissa of at least $-\rho_{0}:=-\min \left(\rho_{01}, \rho_{02}\right)<0$, with

$$
\begin{gathered}
\rho_{01}:=\frac{1}{\left\|A_{0}^{-1}\right\|_{2}}, \\
\rho_{02}:=\frac{1}{\left\|\left(s I_{n}-\sum_{i=0}^{m} A_{i}\right)^{-1}\right\|_{\infty}} \\
=\sup _{s \in \mathbf{C}_{0+}} \bar{\sigma}\left(\left(s I_{n}-\sum_{i=0}^{m} A_{i}\right)^{-1}\right) \\
=\sup _{\omega \in \mathbf{R}_{0+}} \bar{\sigma}\left(\left(\mathbf{i} \omega I_{n}-\sum_{i=0}^{m} A_{i}\right)^{-1}\right),
\end{gathered}
$$

provided that:

(a) $A_{0}$ is a stability matrix,

(b) Equation (4.2) holds for some $\beta_{i} \in \mathbf{R}_{+}(\forall i \in \bar{m})$ such that $\sum_{i=1}^{m} \beta_{i}^{2}=1$

or if $\rho \in\left[0, \kappa_{2}\left(A_{0}\right)\right)$ and, furthermore,

$$
\left\|\left\lfloor\beta_{1}^{-1} A_{1}, \ldots, \beta_{r}^{-1} A_{m}\right\rfloor\right\|_{2}<-\kappa_{2}\left(A_{0}\right)+\rho<0
$$

so that the limiting differential system (4.1) is globally asymptotically stable independent of the delays with stability abscissa of at least $-\kappa_{2}\left(A_{0}\right)<0$.

Proof. (i) Property (i) follows from the results in [4, Proposition 4.8] with (4.2). Note that $h_{i}=0, \forall i \in \bar{m}$, the (delay-free) system (4.1) is globally asymptotically stable so that $\|\left(s I_{n}-\right.$ $\left.\sum_{i=0}^{m} A_{i}\right)^{-1} \|_{\infty}<\infty$ so that $\left\|\left(s I_{n}-\sum_{i=0}^{m} A_{i} e^{-h_{i} s}\right)^{-1}\right\|_{\infty}<\infty$. Then, the limiting differential system associated with (4.1) is globally asymptotically stable independent of the delays if Equation (4.2) holds which is obtained by guaranteeing the inverse below on $\mathbf{C}_{0+}$ under a necessary and sufficiency-type text on the complex imaginary axis:

$$
\left(s I_{n}-\sum_{i=0}^{m} A_{i^{e}}-h_{i^{s}}\right)^{-1}=\left(\sum_{i=0}^{m} A_{i}\left(1-e^{-h_{i s} s}\right)\right)^{-1}\left(s I_{n}-\sum_{i=0}^{m} A_{i}\right)^{-1} .
$$

(ii) Since property (i) holds, then (4.2) holds so that there exist $\beta_{i} \in \mathbf{R}_{+}(\forall i \in \bar{m})$ (which are in general distinct from those in property (i) but we keep the same notation) such that 
$\sum_{i=1}^{m} \beta_{i}^{2}=1$, and for any $\mathbf{R}_{0+} \ni \rho \in\left[0, \rho_{02}\right), \forall \omega \in \mathbf{R}_{0+}$ :

$$
\begin{aligned}
\left\|\left(s I_{n}-\sum_{i=0}^{m} A_{i}\right)\left(I_{n}-\rho\left(s I_{n}-\sum_{i=0}^{m} A_{i}\right)^{-1}\right)^{-1}\left[\beta_{1}^{-1} A_{1}, \ldots, \beta_{r}^{-1} A_{m}\right]\right\|_{\infty} \\
\quad=\left\|\left(\mathbf{i} \omega I_{n}-\sum_{i=0}^{m} A_{i}\right)\left(I_{n}-\rho\left(\mathrm{i} \omega I_{n}-\sum_{i=0}^{m} A_{i}\right)^{-1}\right)^{-1}\left[\beta_{1}^{-1} A_{1}, \ldots, \beta_{r}^{-1} A_{m}\right]\right\|_{2} \\
\quad \leq\left\|\left(\mathbf{i} \omega I_{n}-\sum_{i=0}^{m} A_{i}\right)^{-1}\left[\beta_{1}^{-1} A_{1}, \ldots, \beta_{r}^{-1} A_{m}\right]\right\|_{2}\left(\frac{1}{1-\rho\left\|\left(\mathbf{i} \omega I_{n}-\sum_{i=0}^{m} A_{i}\right)^{-1}\right\|_{2}}\right)<1
\end{aligned}
$$

and the above $H_{\infty}$-norm exists if $\left\|\left(s I_{n}-\sum_{i=0}^{m} A_{i}\right)^{-1}\right\|_{\infty}<\infty$ from Banach Perturbation Lemma, [34]. On the other hand, if $A_{0}$ is stability matrix then $A_{0}+\rho I_{n}$ is still a stability matrix if (4.4) holds by applying the min max computation approach for the eigenvalues of $A_{0}+\rho I_{n}$ which are larger than those of $A_{0}$ since the identity matrix is positive definite. As a result, if global asymptotic stability independent of delays holds then it also holds with stability abscissa $-\rho_{0}<0$. The first part of property (ii) has been proven. The second part follows by replacing (4.3) with

$$
\left\|\left\lfloor\beta_{1}^{-1} A_{1}, \ldots, \beta_{r}^{-1} A_{m}\right\rfloor\right\|_{2}<-\kappa_{2}\left(A_{0}+\rho I_{n}\right) \leq-\kappa_{2}\left(A_{0}\right)+\rho<0 .
$$

Through the use of the properties of the matrix measure $[4,34]$. Theorem 4.1 can be directly combined with Corollaries 3.4-3.5 as follows.

Corollary 4.2. Assume that the functional limiting differential system (4.1) satisfies Theorem 4.1. Thus, both the perturbed and the nominal functional differential systems have a negative strict Lyapunov exponent which satisfies $\mu \leq-\min \left(\rho_{01}, \rho_{02}, \kappa_{2}\left(A_{0}\right)\right)<0$. The solution of $(4.1)$, subject to (2.3) with the hypotheses in Theorem 2.1, and that of its limiting differential system are both globally asymptotically stable independent of the delays.

If, furthermore, $\gamma: \mathbf{R}_{0+} \rightarrow \mathbf{R}_{0+}$ satisfies one of the subsequent conditions:

(a) $\gamma(t)=o\left(e^{-a t}\right)$ as $t \rightarrow \infty$,

(b) $\int_{0}^{\infty} t^{\nu-1} \gamma(t) d t<\infty$ as $t \rightarrow \infty$,

(c) $\gamma(t) \leq o\left(t^{1-v}\right)$ as $t \rightarrow \infty$,

then any solution of the perturbed functional differential system (4.1) fulfils either Corollaries 3.4-3.5 to Theorem 3.2 (under the condition (a)) or Corollary 3.7 to Theorem 3.6 (under the condition (b) or the condition (c)).

A parallel result to Theorem 4.1(i) relies on the subsequent remark. 
Remark 4.3. Note that (4.2) holds if

$$
\begin{aligned}
& \|\left(s I_{n}-A_{0}\right)\left(I_{n}-\left(s I_{n}-A_{0}\right)^{-1}\left(\sum_{i=0}^{m} A_{i}\right)\right)\left(I_{n}-\rho\left(s I_{n}-\sum_{i=0}^{m} A_{i}\right)^{-1}\right)^{-1} \\
& \quad \times\left[\beta_{1}^{-1} A_{1}, \ldots, \beta_{r}^{-1} A_{m}\right] \|_{\infty}<1, \quad \forall s \in \mathbf{C}_{0+}
\end{aligned}
$$

provided that $\left(\left(\mathbf{i} \omega I_{n}-A_{0}\right)\left(I_{n}-\left(\mathbf{i} \omega I_{n}-A_{0}\right)^{-1}\left(\sum_{i=0}^{m} A_{i}\right)\right)\right)^{-1}$ exists, $\omega \in \mathbf{R}_{0+}$. That inverse exists provided that $A_{0}$ is a stability matrix, as it has been assumed, since then it has no imaginary eigenvalues or real ones at the origin and, furthermore,

$$
\begin{gathered}
\left\|\left(s I_{n}-A_{0}\right)^{-1}\left(\sum_{i=1}^{m} A_{i}\right)\right\|_{\infty} \leq a_{0}<1, \\
\rho\left\|\left(s I_{n}-\sum_{i=0}^{m} A_{i}\right)^{-1}\right\|_{\infty} \leq \rho\left\|\left(s I_{n}-A_{0}\right)^{-1}\left(\sum_{i=1}^{m} A_{i}\right)\right\|_{\infty} \leq \rho a_{0} \leq b_{0}<1, \\
\left\|\left(\left(\mathbf{i} \omega I_{n}-A_{0}\right)\left(I_{n}-\left(\mathbf{i} \omega I_{n}-A_{0}\right)^{-1}\left(\sum_{i=0}^{m} A_{i}\right)\right)\right)^{-1}\left[\beta_{1}^{-1} A_{1}, \ldots, \beta_{r}^{-1} A_{m}\right]\right\|_{2}<1, \quad \forall \omega \in \mathbf{R}_{0+}
\end{gathered}
$$

provided that $\left(\left(\mathbf{i} \omega I_{n}-A_{0}\right)\left(I_{n}-\left(\mathbf{i} \omega I_{n}-A_{0}\right)^{-1}\left(\sum_{i=0}^{m} A_{i}\right)\right)\right)^{-1}$ exists, $\forall \omega \in \mathbf{R}_{0+}$. Such an inverse exists provided that $A_{0}$ is a stability matrix, as it has been assumed, since then it has no imaginary eigenvalues or real ones at the origin and, furthermore, $\left\|\left(s I_{n}-A_{0}\right)^{-1}\left(\sum_{i=1}^{m} A_{i}\right)\right\|_{\infty} \leq$ $a_{0}<1$. Then, the system (4.1) is globally asymptotically stable independent of the delays if $\left\|\left(s I_{n}-A_{0}\right)^{-1}\left(\sum_{i=1}^{m} A_{i}\right)\right\|_{\infty} \leq a_{0}<1$ (what implies that $A_{0}$ is a stability matrix) and $\left\|\left[\beta_{1}^{-1} A_{1}, \ldots, \beta_{r}^{-1} A_{m}\right]\right\|_{2}<a_{0}^{-1}$. The most general constraint (4.8) is guaranteed with any $\mathbf{R}_{0+} \ni \rho<a_{0}^{-1}$ if $\left\|\left[\beta_{1}^{-1} A_{1}, \ldots, \beta_{r}^{-1} A_{m}\right]\right\|_{2}<\left(1-p a_{0}\right) / a_{0}$ which guarantees global asymptotic stability independent of the delays of the limiting differential system of (4.1) and $A_{0}$ is a stability matrix of spectral abscissa of at least $-\rho<0$.

\subsection{Functional Differential Equations with Mixed Finite Point and Time-Varying Distributed Delays}

Assume that (2.2)-(2.3) consist of a single finite constant point delay $h_{1} \geq 0$ and a single distributed time-varying one $h:[-1,0] \rightarrow \mathbf{R}_{0+}$ leading to the functional differential system:

$$
\dot{x}(t)=\sum_{i=0}^{1} A_{i} x\left(t-h_{i}\right)+\int_{-1}^{0} d \alpha(\tau) x(t-h(\tau))+f\left(t, x_{t}\right)
$$


with $h_{0}=0$. The perturbation function is defined by (2.3) and satisfies the hypothesis of Theorem 2.1. The limiting equation is defined for identically zero perturbation function. Equation (4.12) is equivalent to:

$$
\begin{aligned}
\dot{x}(t)= & \left(A_{0}+A_{1}+\alpha(0)-\alpha(-1)\right) x(t)+A_{1}\left(x\left(t-h_{1}\right)-x(t)\right) \\
& +\int_{-1}^{0} d \alpha(\tau)(x(t-h(\tau))-x(t))+f\left(t, x_{t}\right),
\end{aligned}
$$

so that the characteristic equation of the limiting equation is:

$$
\begin{aligned}
\operatorname{det} & \left(s I_{n}-A_{0}-A_{1} e^{-h_{1} s}-\int_{-1}^{0} \lambda(\tau) d \alpha(\tau)\right) \\
& =\operatorname{det}\left(s I_{n}-\left(A_{0}+\alpha(0)-\alpha(-1)\right)-A_{1} e^{-h_{1} s}-\int_{-1}^{0} \lambda(\tau) d \alpha(\tau)\right) \\
& =\operatorname{det}\left(s I_{n}-\left(A_{0}+A_{1}+\alpha(0)-\alpha(-1)\right)-A_{1}\left(e^{-h_{1} s}-1\right)-\int_{-1}^{0} \lambda(\tau) d \alpha(\tau)\right)=0,
\end{aligned}
$$

for any continuous function $\lambda(t)$ mapping $[-1,0]$ on the unit circle centred at the origin of the complex plane provided that $\alpha$ is non-atomic at zero. The following result is concerned with the global asymptotic stability of the differential system (4.12).

Theorem 4.4. The following properties hold.

(i) Assume that $A_{0}$ is a stability matrices, so that $\exists \rho_{1} \in \mathbf{R}_{+}: \kappa_{2}\left(A_{0}\right) \leq-\rho_{1}<0$. Thus, if

$$
\frac{1}{\left\|A_{1}\right\|_{2}+\left\|\int_{-1}^{0} \lambda(\tau) d \alpha(\tau)\right\|_{2}}\left\|\left(s I_{n}-A_{0}-\rho_{1} I_{n}\right)^{-1}\right\|_{\infty}<1
$$

then the limiting equation associated with (4.12) is globally asymptotically stable independent of the delays.

(ii) Assume that $A_{0}$ and $\left(A_{0}+\alpha(0)-\alpha(-1)\right)$ are both stability matrices, so that $\exists \rho_{2} \in \mathbf{R}_{+}$: $\kappa_{2}\left(A_{0}+\alpha(0)-\alpha(-1)\right) \leq-\rho_{2}<0$. Thus, if

$$
\frac{1}{\left\|A_{1}\right\|_{2}+\|\alpha(0)\|_{2}+\|\alpha(1)\|_{2}}\left\|\left(s I_{n}-A_{0}-\alpha(0)+\alpha(-1)-\rho_{2} I_{n}\right)^{-1}\right\|_{\infty}<1,
$$

then the limiting equation associated with (4.12) is globally asymptotically stable independent of the delays. 
(iii) Assume that $A_{0}+A_{1}+\alpha(0)-\alpha(-1)$ is stability matrix so that $\exists \rho_{3} \in \mathbf{R}_{+}: \kappa_{2}\left(A_{0}+A_{1}+\right.$ $\alpha(0)-\alpha(-1)) \leq-\rho_{3}<0$. Thus, if

$$
\frac{1}{2\left\|A_{1}\right\|_{2}+\|\alpha(0)\|_{2}+\|\alpha(1)\|_{2}}\left\|\left(s I_{n}-A_{0}-A_{1}-\alpha(0)+\alpha(-1)-\rho_{3} I_{n}\right)^{-1}\right\|_{\infty}<1,
$$

then the limiting equation associated with (4.12) is globally asymptotically stable independent of the delays.

Proof. Property (i) is direct by using a similar reasoning to that in Theorem 4.1 by extending a result in [4, Section 4.4.5] for a single distributed delayed dynamics, the limiting differential system associated with (4.12) is globally asymptotically stable if and only if for any noncharacteristic zero of the limiting equation, $\operatorname{det}\left(s I_{n}-A_{0}-A_{1} e^{-h s}-\int_{-1}^{0} \lambda(\tau) d \alpha(\tau)\right) \neq 0$, $\forall s \in \mathbf{C}_{0+}$, or equivalently, if the inverse below exists for $\forall s \in \mathbf{C}_{0+}$ by using the rearrangement (4.14),

$$
\begin{aligned}
\left(s I_{n}\right. & \left.-A_{0}-A_{1} e^{-h s}-\int_{-1}^{0} \lambda(\tau) d \alpha(\tau)\right)^{-1} \\
& =\left(\left(s I_{n}-A_{0}-\rho_{1} I_{n}\right)\left(I_{n}-\left(s I_{n}-A_{0}-\rho_{1} I_{n}\right)^{-1}\left(A_{1} e^{-h s}+\int_{-1}^{0} \lambda(\tau) d \alpha(\tau)\right)\right)^{-1}\right) .
\end{aligned}
$$

Such an inverse exists within $\mathbf{C}_{0+}$ under the conditions in property (i) so that the evolution operator exists $T(t, 0)$ since its associate resolvent exists and it is compact in $\mathbf{C}_{0+}$. Properties (ii)-(iii) are direct alternative sufficient conditions to those involved in property (i) by using similar rearrangements for an inverse matrix as (4.20) by using (4.15) and (4.16), respectively, instead of (4.14).

A parallel result to Corollary 4.2 now follows from Theorem 4.4.

Corollary 4.5. Assume that the functional limiting differential system of (4.12) satisfies any of the Properties of Theorem 4.4. Thus, both the limiting and the perturbed functional differential equation have a negative strict Lyapunov exponent satisfying $\mu \leq-\rho<0$ with $\rho$ equalizing the corresponding $\rho_{i}(i=1,2,3)$. Corollary 4.2 holds "mutatis-mutandis."

The extension of Theorem 4.4 and Corollary 4.5 to the case of multiple point and distributed delays is direct and then omitted.

\section{Example}

Consider the second-order linear functional equation with point time-delay $h$ :

$$
\begin{aligned}
\ddot{x}(t) & =-\alpha(t) \dot{x}(t)-\beta(t) \dot{x}(t-h)-\gamma(t) x(t)-\omega(t) x(t-h) \\
& =-\alpha_{0} \dot{x}(t)-\beta_{0} \dot{x}(t-h)-\gamma_{0} x(t)-\omega_{0} x(t-h)+f\left(t, x_{t}\right)
\end{aligned}
$$


subject to arbitrary bounded initial conditions within the proposed class, where the disturbance is

$$
f\left(t, x_{t}\right)=\left(\alpha_{0}-\alpha(t)\right) \dot{x}(t)+\left(\beta_{0}-\beta(t)\right) \dot{x}(t-h)+\left(\gamma_{0}-\gamma(t)\right) x(t)+\left(\omega_{0}-\omega(t)\right) x(t-h),
$$

and the limiting equation is

$$
\ddot{y}(t)=-\alpha_{0} \dot{y}(t)-\beta_{0} \dot{y}(t-h)+\gamma_{0} y(t)-\omega_{0} y(t-h),
$$

provided that $\alpha(t) \rightarrow \alpha_{0}, \beta(t) \rightarrow \beta_{0}, \gamma(t) \rightarrow \gamma_{0}$, and $\omega(t) \rightarrow \omega_{0}$ as $t \rightarrow \infty$. Note that the perturbation term (5.2) satisfies (2.8) of Theorem 2.1. The differential equation and limiting differential equation may be rewritten equivalently as the following second-order dynamic systems of respective state vectors $z_{x}(t)=\left(x_{1}(t), x_{2}(t)\right)^{T}$ and $z_{y}(t)=\left(y_{1}(t), y_{2}(t)\right)^{T}$,

$$
\begin{aligned}
\dot{z}_{x}(t) & =A_{0 y} z_{x}(t)+A_{1 y} z_{x}(t-h)+z_{f}\left(t, z_{x t}\right) \\
& =A_{0}(t) z_{x}(t)+A_{1}(t) z_{x}(t-h), \\
\dot{z}_{y}(t) & =A_{0 y} z_{y}(t)+A_{1 y} z_{y}(t-h),
\end{aligned}
$$

where

$$
\begin{gathered}
A_{0 y}=\left[\begin{array}{cc}
1 & 1 \\
-\gamma_{0} & -\alpha_{0}
\end{array}\right] ; \quad A_{1 y}=\left[\begin{array}{cc}
1 & 1 \\
-\omega_{0} & -\beta_{0}
\end{array}\right] ; \quad z_{f}\left(t, z_{x t}\right)=\left[\begin{array}{c}
0 \\
f\left(t, x_{t}\right)
\end{array}\right], \\
A_{0}(t)=\left[\begin{array}{cc}
1 & 1 \\
-\gamma(t) & -\alpha(t)
\end{array}\right] ; \quad A_{1}(t)=\left[\begin{array}{cc}
1 & 1 \\
\omega(t) & -\beta(t)
\end{array}\right] .
\end{gathered}
$$

The eigenvalues of $A_{0 y}$ are $\alpha_{1,2}=\left(-\alpha_{0} \mp \sqrt{\alpha_{0}^{2}-4 \gamma_{0}}\right) / 2$ so that $A_{0 y}$ is a stability matrix if $\alpha_{0}>0$ and $\gamma_{0}>0$. The limiting equation (5.3) is globally stable independent of the delay size if $\kappa_{2}\left(T^{-1} A_{0 y} T\right)<\left\|T^{-1} A_{1 y} T\right\|_{2}$ for at least one second-order nonsingular state transformation matrix $T$, where $\kappa_{2}(\cdot)$ and $\|\cdot\|_{2}$ are the 2-matrix measure with respect to the $\ell_{2}$-norm, [4], and $\ell_{2}$-norm of the matrix $(\cdot)$, respectively. The use of a similarity transformation is usually necessary by the fact that the matrix measure is norm-dependent and it can be positive even for stability matrices for some norms and the stability property is independent of any performed similarity transformation on the state variables in the linear time- invariant case, $[4,5,11]$. Now, choose as state transformation matrix the nonsingular Vandermonde matrix $T=\left[\begin{array}{cc}1 & 1 \\ \alpha_{1} & \alpha_{2}\end{array}\right]$ which defines the diagonal stability matrix $A_{0 y d}=T^{-1} A_{0 y} T$, being similar to $A_{0 y}$, which possesses a negative 2-matrix measure $\kappa_{2}\left(A_{0 y d}\right)=-\alpha_{0}<0$. In this case,

$$
A_{1 y d}=T^{-1} A_{1 y} T=\frac{1}{\alpha_{2}-\alpha_{1}}\left[\begin{array}{ll}
\omega_{0}+\beta_{0} \alpha_{1} & \omega_{0}+\beta_{0} \alpha_{2} \\
\omega_{0}+\beta_{0} \alpha_{1} & \omega_{0}+\beta_{0} \alpha_{2}
\end{array}\right],
$$


and the limiting equation is globally asymptotically Lyapunov stable independent of the delay size $h$ if

$$
\begin{aligned}
\left|\mathcal{K}_{2}\left(A_{0 y d}\right)\right| & =\alpha_{0}>\left\|A_{1 y d}\right\|_{2} \\
& =\sqrt{\lambda_{\max }\left(A_{1 y d}^{T} A_{1 y d}\right)} \\
& =\frac{\left(2+\beta_{0}\left(\alpha_{2}-\alpha_{1}\right)\right)\left|\omega_{0}+\beta_{0} \alpha_{1}\right|}{\alpha_{2}-\alpha_{1}} \\
& =\left(\beta_{0}+\frac{2}{\sqrt{\alpha_{0}^{2}-4 \gamma_{0}}}\right)\left|\omega_{0}-\frac{\beta_{0}\left(\alpha_{0}+\sqrt{\alpha_{0}^{2}-4 \gamma_{0}}\right)}{2}\right|,
\end{aligned}
$$

where $\lambda_{\max }(\cdot)$ denotes the maximum eigenvalue of the $(\cdot)$-positive semidefinite matrix. From (5.7), the limiting equation (5.3) is globally asymptotically stable independent of the delay size if $\alpha_{0} \geq 4 \gamma_{0}, \gamma_{0}>0$, and either

$$
\omega_{0}>\frac{1}{2} \max \left(\alpha_{0}\left(\beta_{0}+\sqrt{\alpha_{0}^{2}-4 \gamma_{0}}\right), \beta_{0}\left(\alpha_{0}+\sqrt{\alpha_{0}^{2}-4 \gamma_{0}}\right)\right)
$$

or

$$
\frac{\beta_{0}\left(\alpha_{0}+\sqrt{\alpha_{0}^{2}-4 \gamma_{0}}\right)}{2} \geq \omega_{0}>\frac{2 \beta_{0}-\alpha_{0}}{2} \sqrt{\alpha_{0}^{2}-4 \gamma_{0}}
$$

the second one provided that $\beta_{0} \alpha_{0}+\left(\alpha_{0}-\beta_{0}\right) \sqrt{\alpha_{0}^{2}-4 \gamma_{0}}>0$. Consider the following two cases.

(1) Assume $\alpha(t)=\alpha_{0} ; \forall t \in \mathbf{R}_{0+}$. For some chosen finite $T_{0} \in \mathbf{R}_{0+}$, define:

$$
\begin{array}{cl}
\bar{\beta}\left(T_{0}\right):=\max _{t \geq T_{0}}|\tilde{\beta}(t)| ; & \bar{\gamma}\left(T_{0}\right):=\max _{t \geq T_{0}}|\tilde{\gamma}(t)| ; \quad \bar{\omega}\left(T_{0}\right):=\max _{t \geq T_{0}}|\tilde{\omega}(t)|, \\
\tilde{\beta}(t):=\beta(t)-\beta_{0} ; & \tilde{\gamma}(t):=\gamma(t)-\gamma_{0} ; \quad \tilde{\omega}(t):=\omega(t)-\omega_{0} .
\end{array}
$$

The three above functions converge asymptotically to zero as time tends to infinity. Then, the solution of the differential equation (5.1), subject to (5.2), converges asymptotically to that of its limiting equation (5.3) if $\alpha_{0}^{2} \geq \gamma_{0}+\bar{\gamma}\left(T_{0}\right)$ and (5.8) is replaced with

$$
\begin{aligned}
& \omega_{0}>\bar{\omega}\left(T_{0}\right)+\frac{1}{2} \max ( \alpha_{0}\left(\beta_{0}+\bar{\beta}\left(T_{0}\right)+\sqrt{\alpha_{0}^{2}-4\left(\gamma_{0}-\bar{\gamma}\left(T_{0}\right)\right)}\right), \\
&\left.\left(\beta_{0}+\bar{\beta}\left(T_{0}\right)\right)\left(\alpha_{0}+\sqrt{\alpha_{0}-4\left(\gamma_{0}-\bar{\gamma}\left(T_{0}\right)\right)}\right)\right) .
\end{aligned}
$$


Another convergence condition of the solution of (5.2)-(5.3) to the limiting solution can be directly obtained from (5.9). The limiting differential equation (5.3) has a dominant eigenvalue $\bar{\lambda}$ of real part:

$$
0>\operatorname{Re} \overline{\mathcal{\lambda}}=\mu \geq \kappa_{2}\left(A_{0 d}\right)-\left\|A_{1 d}\right\|_{2}-\max _{t \geq T_{0}}\left\|\tilde{A}_{0 d}(t)\right\|_{2}-\max _{t \geq T_{0}}\left\|\tilde{A}_{1 d}(t)\right\|_{2}
$$

with $K_{0 \alpha}>0$ related to the perturbation (5.3), so that $\Lambda \neq \varnothing$ according to (2.20) and Theorem 2.3(i2), and the strict Lyapunov exponent $\mu$ defined in Theorem 2.3 satisfying the above lower-bound, and

$$
\tilde{A}_{0 d}(t)=T^{-1}\left[\begin{array}{cc}
0 & 0 \\
-\tilde{\gamma}(t) & 0
\end{array}\right] T, \quad \tilde{A}_{1 d}(t)=T^{-1}\left[\begin{array}{cc}
0 & 0 \\
-\tilde{\omega}(t) & -\tilde{\beta}(t)
\end{array}\right] T ; \quad \forall t \in \mathbf{R}_{0+} .
$$

(2) Now, assume $\beta(t)=\beta_{0}, \gamma(t)=\gamma_{0}, \omega(t)=\omega_{0} ; \forall t \in \mathbf{R}_{0+}$, and $\bar{\alpha}\left(T_{0}\right):=\max _{t \geq T_{0}} \mid \alpha(t)-$ $\alpha_{0} \mid ; \forall t \in \mathbf{R}_{0+}$. Then, the solution of the differential equation (5.1), subject to (5.2), converges asymptotically to that of its limiting equation (5.3) independent of the delay size if $\alpha_{0}^{2} \geq$ $\gamma_{0}+\bar{\gamma}\left(T_{0}\right)$ and (5.8) is replaced with

$$
\begin{gathered}
\omega_{0}>\frac{1}{2} \max \left(\left(\alpha_{0}+\bar{\alpha}\left(T_{0}\right)\right)\left(\beta_{0}+\sqrt{\left(\alpha_{0}+\bar{\alpha}\left(T_{0}\right)\right)^{2}-4 \gamma_{0}}\right),\right. \\
\left.\beta_{0}\left(\alpha_{0}+\sqrt{\left(\alpha_{0}+\bar{\alpha}\left(T_{0}\right)\right)^{2}-4 \gamma_{0}}\right)\right) .
\end{gathered}
$$

In this case, $0>\operatorname{Re} \bar{\lambda}=\mu \geq \kappa_{2}\left(A_{0 d}\right)-\left\|A_{1 d}\right\|_{2}-\bar{\alpha}\left(T_{0}\right)$. Another convergence condition independent of the delay size can easily be got from (5.9). Conditions for asymptotic convergence of the solution to that of its limiting differential equation for the case of complex eigenvalues can be obtained straightforwardly.

\section{Acknowledgments}

The author is very grateful to the Spanish Ministry of Education for its partial support of this work through the project DPI2006-00714. He is also grateful to the Basque Government for its support through GIC07143-IT-269-07, SAIOTEK SPED06UN10, and SPE07UN04. The author is very grateful to the reviewers for their interesting comments which helped him to improve the first version of the manuscript.

\section{References}

[1] M. De la Sen and N. Luo, "Discretization and FIR filtering of continuous linear systems with internal and external point delays," International Journal of Control, vol. 60, no. 6, pp. 1223-1246, 1994.

[2] T. A. Burton, Stability and Periodic Solutions of Ordinary and Differential Equations, vol. 178 of Mathematics in Science and Engineering, Academic Press, Orlando, Fla, USA, 1985.

[3] J. K. Hale and S. M. Verduyn Lunel, Introduction to Functional Differential Equations, vol. 99 of Applied Mathematical Sciences, Springer, New York, NY, USA, 1993.

[4] S.-I. Niculescu, Delay Effects on Stability. A Robust Control Approach, vol. 269 of Lecture Notes in Control and Information Sciences, Edited by M. Thoma, M. Morari, Springer, Berlin, Germany, 2001. 
[5] M. De la Sen and N. Luo, "On the uniform exponential stability of a wide class of linear time-delay systems," Journal of Mathematical Analysis and Applications, vol. 289, no. 2, pp. 456-476, 2004.

[6] M. De la Sen, "Stability of impulsive time-varying systems and compactness of the operators mapping the input space into the state and output spaces," Journal of Mathematical Analysis and Applications, vol. 321, no. 2, pp. 621-650, 2006.

[7] R. F. Datko, "Time-delayed perturbations and robust stability," in Differential Equations, Dynamical Systems and Control Science, vol. 152 of Lecture Notes in Pure ands Applied Mathematics, pp. 457-468, Marcel Dekker, New York, NY, USA, 1994.

[8] C. F. Alastruey, M. De la Sen, and J. R. González de Mendívil, "The stabilizability of integrodifferential systems with two distributed delays," Mathematical and Computer Modelling, vol. 21, no. 8, pp. 85-94, 1995.

[9] N. U. Ahmed, "Optimal control of infinite-dimensional systems governed by integro differential equations," in Differential Equations, Dynamical Systems and Control Science, vol. 152 of Lecture Notes in Pure and Applied Mathematics, pp. 383-402, Marcel Dekker, New York, NY, USA, 1994.

[10] M. R. S. Kulenović, G. Ladas, and A. Meimaridou, "Stability of solutions of linear delay differential equations," Proceedings of the American Mathematical Society, vol. 100, no. 3, pp. 433-441, 1987.

[11] M. De la Sen, “On impulsive time-varying systems with unbounded time-varying point delays: stability and compactness of the relevant operators mapping the input space into the state and output spaces," The Rocky Mountain Journal of Mathematics, vol. 37, no. 1, pp. 79-129, 2007.

[12] Y. Jiang and Y. Jurang, "Positive solutions and asymptotic behavior of delay differential equations with nonlinear impulses," Journal of Mathematical Analysis and Applications, vol. 207, no. 2, pp. 388396, 1997.

[13] T. Sengadir, "Asymptotic stability of nonlinear functional differential equations," Nonlinear Analysis: Theory, Methods E Applications. Series A, vol. 28, no. 12, pp. 1997-2003, 1997.

[14] M. De la Sen, "An algebraic method for pole placement in multivariable systems with internal and external point delays by using single rate or multirate sampling," Dynamics and Control, vol. 10, no. 1, pp. 5-31, 2000.

[15] M. De la Sen, “On positivity of singular regular linear time-delay time-invariant systems subject to multiple internal and external incommensurate point delays," Applied Mathematics and Computation, vol. 190, no. 1, pp. 382-401, 2007.

[16] F. Chen and C. Shi, "Global attractivity in an almost periodic multi-species nonlinear ecological model," Applied Mathematics and Computation, vol. 180, no. 1, pp. 376-392, 2006.

[17] X. Meng, W. Xu, and L. Chen, "Profitless delays for a nonautonomous Lotka-Volterra predator-prey almost periodic system with dispersion," Applied Mathematics and Computation, vol. 188, no. 1, pp. 365-378, 2007.

[18] L. White, F. White, Y. Luo, and T. Xu, "Estimation of parameters in carbon sequestration models from net ecosystem exchange data," Applied Mathematics and Computation, vol. 181, no. 2, pp. 864-879, 2006.

[19] R. R. Sarkar, B. Mukhopadhyay, R. Bhattacharyya, and S. Banerjee, "Time lags can control algal bloom in two harmful phytoplankton-zooplankton system," Applied Mathematics and Computation, vol. 186, no. 1, pp. 445-459, 2007.

[20] L. Bakule and J. M. Rossell, "Overlapping controllers for uncertain delay continuous-time systems," Kybernetika, vol. 44, no. 1, pp. 17-34, 2008.

[21] X. Xiang and N. U. Ahmed, "Existence of periodic solutions of semilinear evolution equations with time lags," Nonlinear Analysis: Theory, Methods E Applications. Series A, vol. 18, no. 11, pp. 1063-1070, 1992.

[22] J. Wang, X. Xiang, and W. Wei, "Linear impulsive periodic system with time-varying generating operators on Banach space," Advances in Difference Equations, vol. 2007, Article ID 26196, 16 pages, 2007.

[23] J. Wang, X. Xiang, W. Wei, and Q. Chen, "Existence and global asymptotical stability of periodic solution for the $T$-periodic logistic system with time-varying generating operators and $T_{0}$-periodic impulsive perturbations on Banach spaces," Discrete Dynamics in Nature and Society, vol. 2008, Article ID 524945, 16 pages, 2008.

[24] J. Wang, X. Xiang, and W. Wei, "Periodic solutions of semilinear impulsive periodic system with time-varying generating operators on Banach space," Mathematical Problems in Engineering, vol. 2008, Article ID 183489, 15 pages, 2008. 
[25] B. Sasu, "Robust stability and stability radius for variational control systems," Abstract and Applied Analysis, vol. 2008, Article ID 381791, 29 pages, 2008.

[26] G. Grammel, "Homogenization of elliptic differential equations in one-dimensional spaces," Abstract and Applied Analysis, vol. 2007, Article ID 98538, 6 pages, 2007.

[27] W. A. Coppel, Stability and Asymptotic Behavior of Differential Equations, Heath, Boston, Mass, USA, 1965.

[28] M. Pituk, "Asymptotic behavior and oscillation of functional differential equations," Journal of Mathematical Analysis and Applications, vol. 322, no. 2, pp. 1140-1158, 2006.

[29] M. Pituk, "A Perron type theorem for functional differential equations," Journal of Mathematical Analysis and Applications, vol. 316, no. 1, pp. 24-41, 2006.

[30] G. Da Prato and M. Iannelli, "Linear integro-differential equations in Banach spaces," Rendiconti del Seminario Matematico dell'Università di Padova, vol. 62, pp. 207-219, 1980.

[31] N. Dunford and J. T. Schwartz, Linear Operators. Part I, John Wiley \& Sons, New York, NY, USA, 1958.

[32] S. Barnett, Polynomials and Linear Control Systems, vol. 77 of Monographs and Textbooks in Pure and Applied Mathematics, Marcel Dekker, New York, NY, USA, 1983.

[33] U. Mackenroth, Robust Control Systems. Theory and Case Studies, Springer, Berlin, Germany, 2004.

[34] D. S. Watkins, The Matrix Eigenvalue Problem. GR and Krylov Subspace Methods, SIAM, Philadelphia, Pa, USA, 2007. 


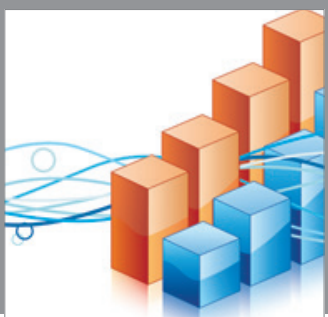

Advances in

Operations Research

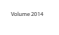

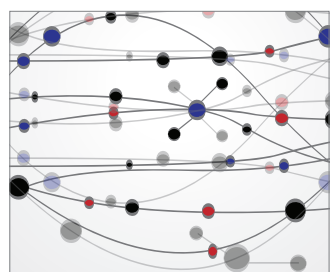

\section{The Scientific} World Journal
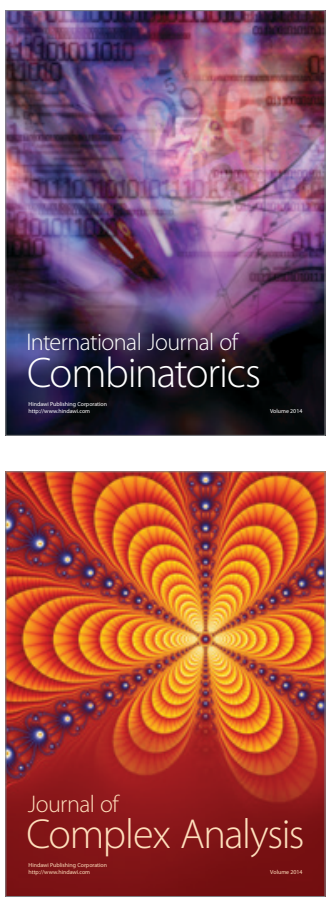

International Journal of

Mathematics and

Mathematical

Sciences
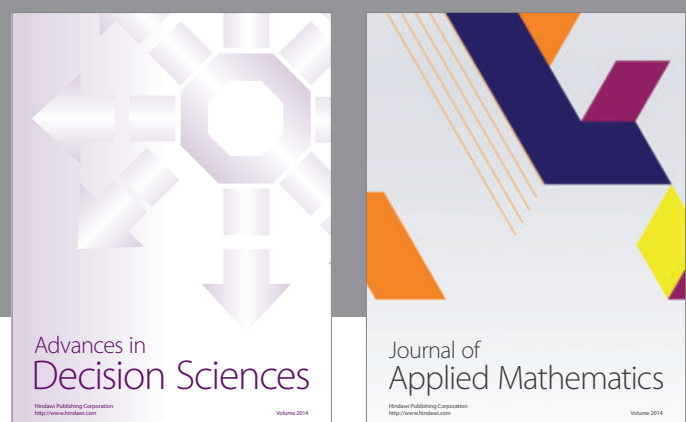

Journal of

Applied Mathematics
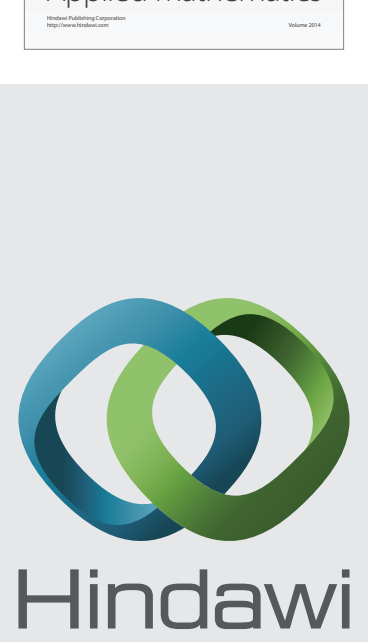

Submit your manuscripts at http://www.hindawi.com
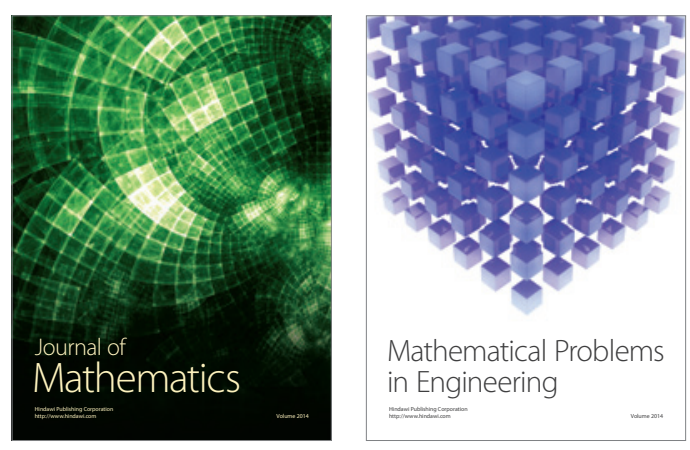

Mathematical Problems in Engineering
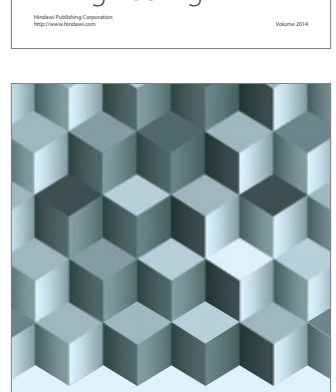

Journal of

Function Spaces
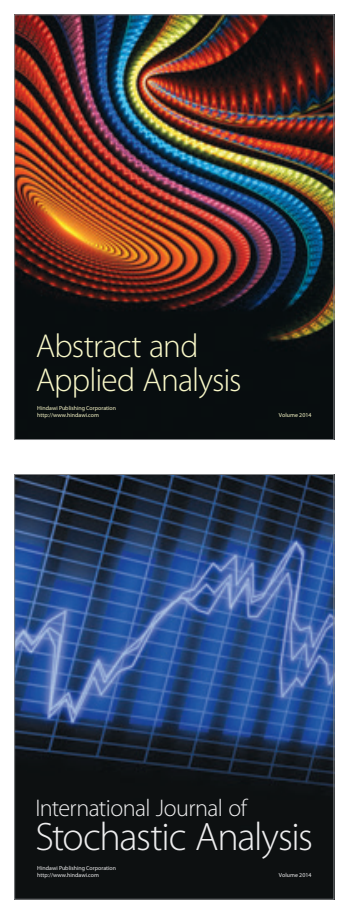

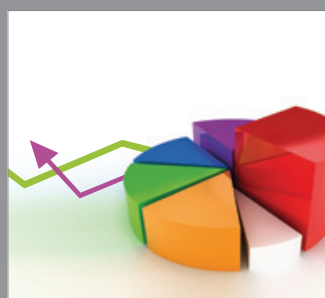

ournal of

Probability and Statistics

Promensencen
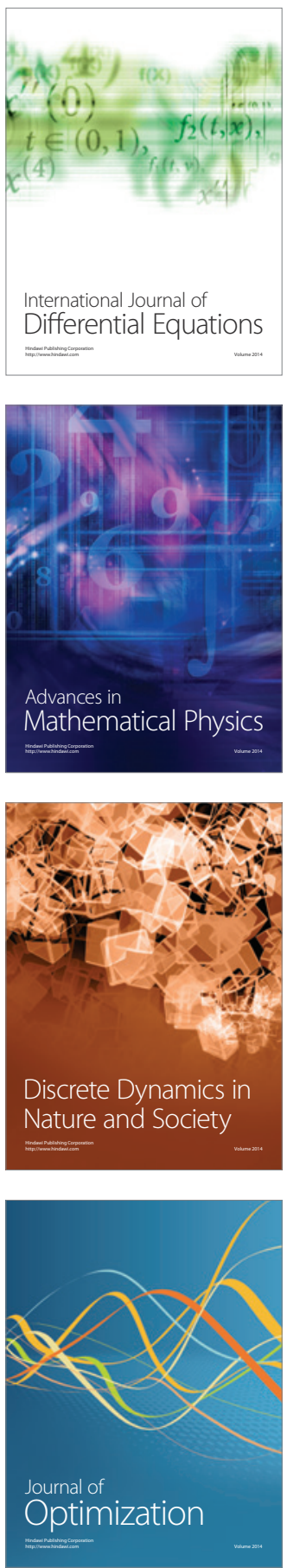